\title{
Role of the Dietitian in Multidisciplinary Treatment of Polycystic Ovary Syndrome
}

Wendy Thompson

West Virginia University

Follow this and additional works at: https://researchrepository.wvu.edu/etd

\section{Recommended Citation}

Thompson, Wendy, "Role of the Dietitian in Multidisciplinary Treatment of Polycystic Ovary Syndrome" (2014). Graduate Theses, Dissertations, and Problem Reports. 547.

https://researchrepository.wvu.edu/etd/547

This Thesis is protected by copyright and/or related rights. It has been brought to you by the The Research Repository @ WVU with permission from the rights-holder(s). You are free to use this Thesis in any way that is permitted by the copyright and related rights legislation that applies to your use. For other uses you must obtain permission from the rights-holder(s) directly, unless additional rights are indicated by a Creative Commons license in the record and/ or on the work itself. This Thesis has been accepted for inclusion in WVU Graduate Theses, Dissertations, and Problem Reports collection by an authorized administrator of The Research Repository @ WVU. For more information, please contact researchrepository@mail.wvu.edu. 


\author{
Role of the Dietitian in \\ Multidisciplinary Treatment of Polycystic Ovary Syndrome \\ Wendy Thompson \\ Thesis submitted to the \\ Davis College of Agriculture, Natural Resources and Design \\ at \\ West Virginia University \\ in partial fulfillment of the requirements \\ for the degree \\ of \\ Master of Science \\ in \\ Human Nutrition
}

Approved by

Melissa D. Olfert, DrPH, MS, RD, LD, Committee Chairperson

Pamela J. Murray, MD, MHP

Melanie Clemmer, $\mathrm{PhD}$

Department of Animal and Nutritional Sciences

Morgantown, West Virginia

2014

Keywords: Polycystic Ovary Syndrome, PCOS, Multidisciplinary, Registered Dietitian, Nutrition Interventions

Copyright 2014 Wendy Thompson 


\begin{abstract}
Role of the Dietitian in Multidisciplinary Treatment of Polycystic Ovary Syndrome

Wendy Thompson
\end{abstract}

Background: Polycystic Ovary Syndrome (PCOS) is the most common reproductive endocrine disorder in females. Genetic and lifestyle factors influence the etiology and insulin resistance plays a key role in the pathogenesis of PCOS.

Objective: To investigate the current trends and future implications of multidisciplinary PCOS clinics while emphasizing the role and challenges for dietitians.

Methods: The study design was a two-phase formative investigation of PCOS focused practitioners through an anonymous, internet-based survey (Qualtrics, Provo, UT) followed by focus groups done via teleconference. Focus group data was analyzed using Braun and Clarke's method of thematic analysis.

Participants: Survey respondents included 261 health care providers, 59\% physicians, $20 \%$ dietitians, from around the world (64\% from the United States); the majority (59\%) represented multidisciplinary facilities. Focus group participants included four dietitians, three physicians, a health psychologist and a licensed nutritionist that had 7-25 years of experience treating PCOS.

Results: From the survey, the barriers for future multidisciplinary clinics included: money/resources, insurance reimbursement, and difference of opinions; the potential advantages included: more comprehensive and integrated care, greater convenience/efficiency, better longterm outcomes, and increased access to disciplines. Dietitians were involved in $71 \%$ of the clinics represented in the survey and $89 \%$ of respondents stated that dietitians need to be 'involved' or 'highly involved' in PCOS treatment. Focus group participants stated the greatest challenges for dietitians include insurance, lack of PCOS knowledge, and lack of physician referrals. Overall, nutritional interventions are not very accessible for the majority of PCOS patients.

Conclusions and Implications: PCOS is a complex condition that requires the expertise of multiple provider types to treat the syndrome in its entirety. Most providers agreed that multidisciplinary clinics would ultimately lead to a better prognosis for PCOS patients. A greater emphasis needs to be placed on educating the medical community, including dietitians and physicians, on the importance of specialized nutrition counseling and lobbying for insurance reimbursement. Having access to dietitians educated on PCOS is likely the best way to ensure that PCOS patients have access to lifestyle interventions, which is considered to be the first-line treatment for PCOS. 


\section{Acknowledgments}

There are a number of people whom I would like to genuinely thank for their contributions, assistance, and support over the course of the past two years. First of all, I want to extend my upmost of gratitude to Dr. Melissa Olfert, my thesis advisor and dietetic internship director, because without her insightful guidance I would have never been presented with the basis of this idea for my research. Dr. Olfert has fully supported me and pushed me towards new challenges over these past two years. I am also thankful for my wonderful committee members, Dr. Pamela Murray and Dr. Melanie Clemmer, who dedicated their time and efforts to assisting me with recruitment and the development of this thesis. They have been nothing but supportive and have provided great insight towards this project and my future career. I would also like to mention my thanks for Mackenzie Barr and Jade White for assisting me with their fantastic notetaking skills.

I would like to acknowledge all the participates that dedicated their time towards my survey and I am sincerely appreciative of the individuals that donated their time to engage in my focus groups; without you all my study would not have been successful.

I owe my highest gratitude for my family and closest friends because without your ongoing support this endeavor would not have been possible. To my parents, you have always believed in me and provided me the encouragement I have needed to succeed and continue through all I have chosen to conquer in life. To Kyle, you have always allowed me to take on new challenges, even if that meant moving across the country. Your support, love, and sense of humor have kept me going through the exams, papers, and countless presentations. Thank you all for everything you have done to help me succeed. 


\section{Table Of Contents}

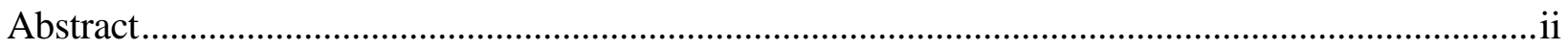

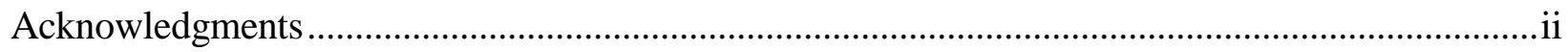

Table Of Contents ……………………............................................................................................

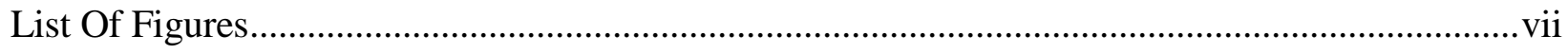

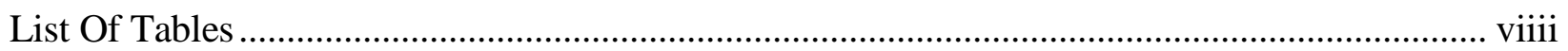

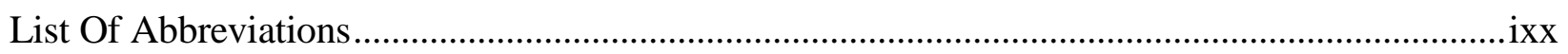

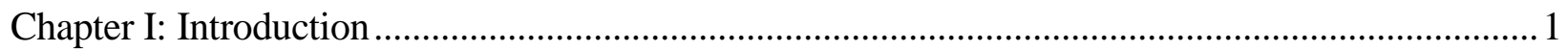

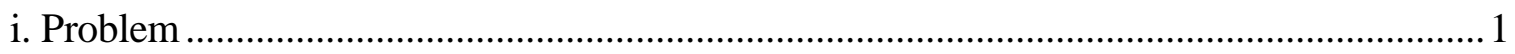

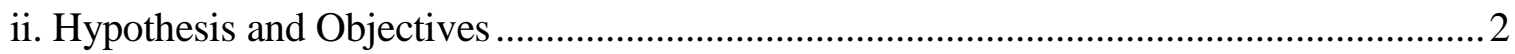

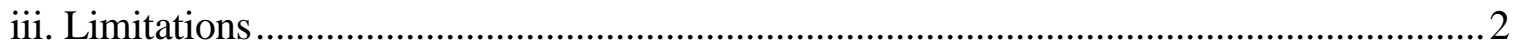

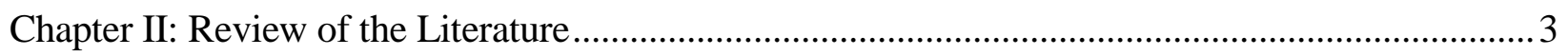

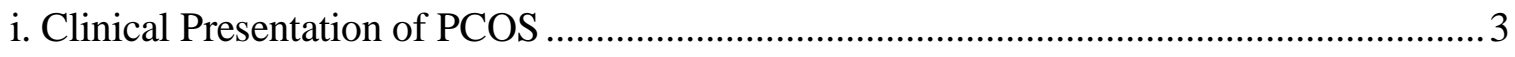

ii. Geographical Prevalence of PCOS ...........................................................................

Understanding the Prevalence of PCOS ................................................................. 4

Effect of the Diagnostic Criteria on Prevalence …………………………………..... 6

Prevalence of PCOS Across the US ………………………….................................

Prevalence of PCOS in Caucasians Across the World .............................................. 8

Prevalence of PCOS Across Different Races/Ethnicities ............................................ 9

PCOS Prevalence Conclusion ........................................................................... 13

iii. The Current Description and Future Need for Multidisciplinary PCOS Clinics .............14

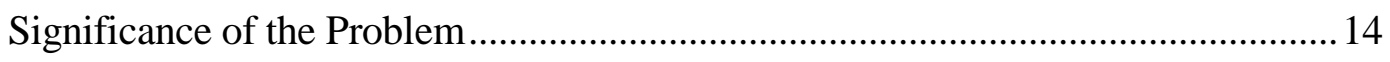

Efficiacy of Multidisciplinary PCOS Clinics ............................................................. 17

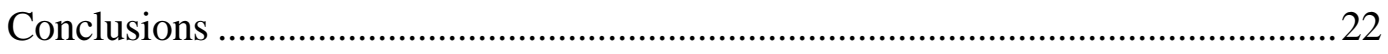

iv. Lifestyle Interventions and the Role of Dietitians in the Treatment of PCOS ................23 
The Role of Registered Dietitians in the Treatment of PCOS ................................24

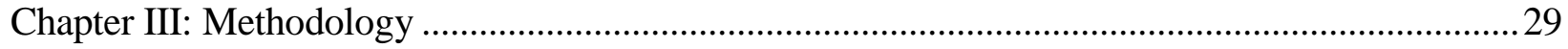

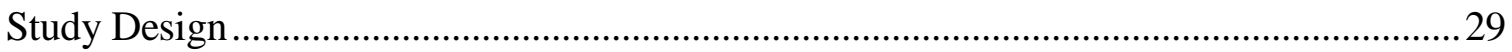

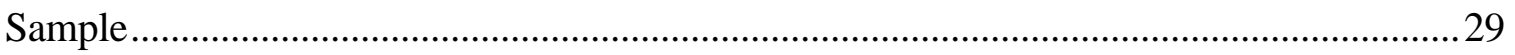

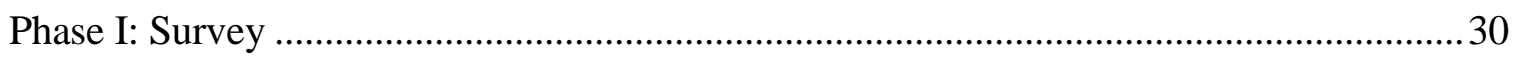

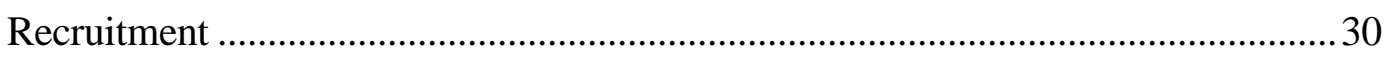

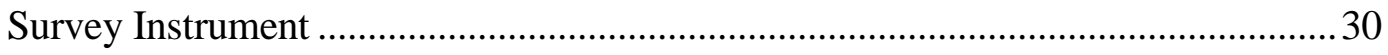

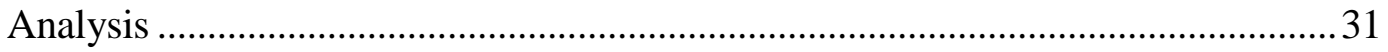

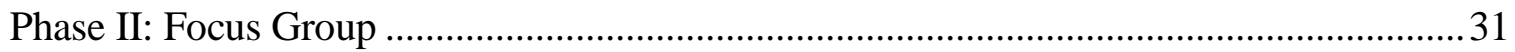

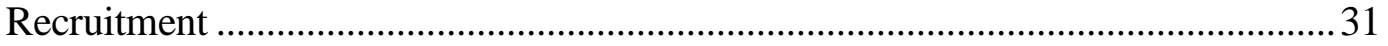

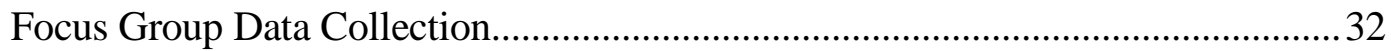

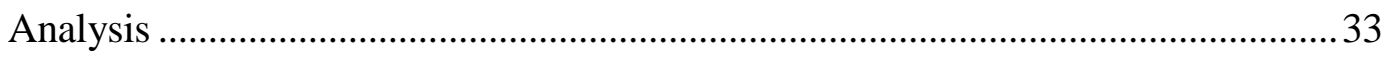

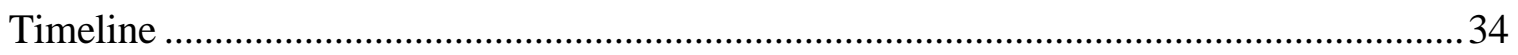

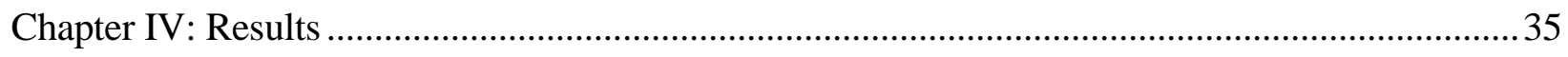

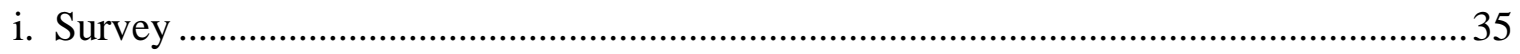

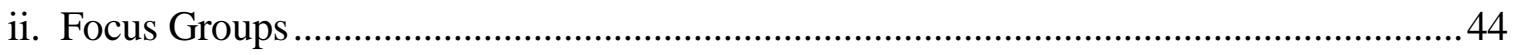

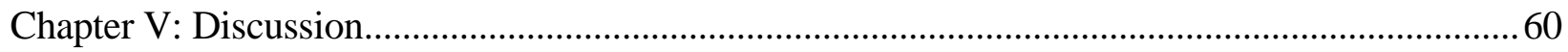

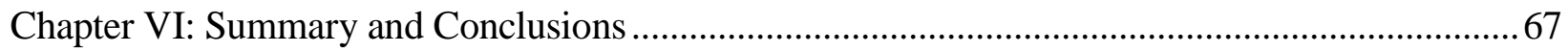

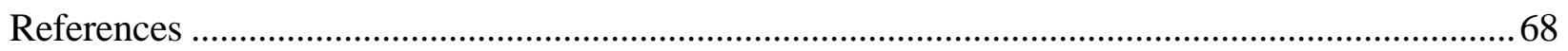

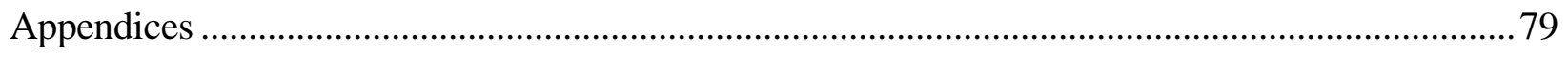

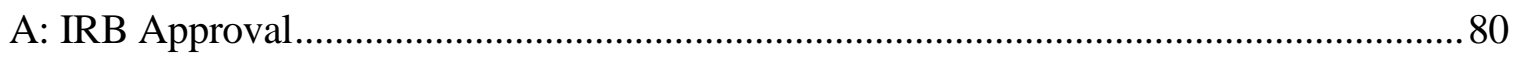

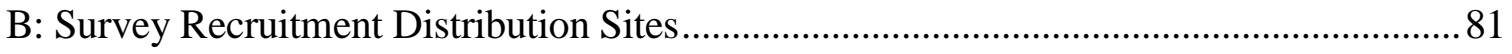

C: Survey Announcement for List Serves and LinkedIn................................................... 82

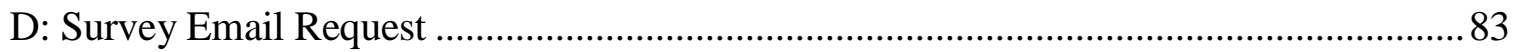


E: Survey Email Reminder.

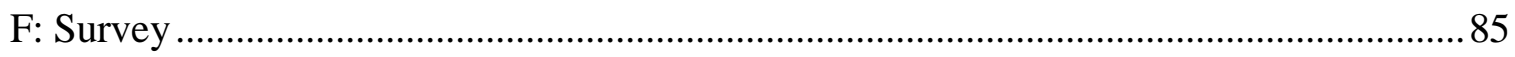

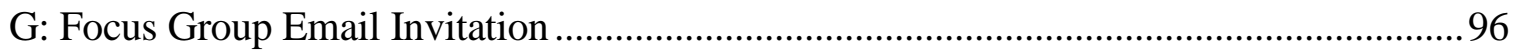

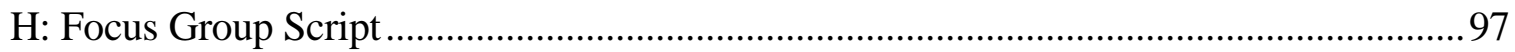




\section{List of Figures}

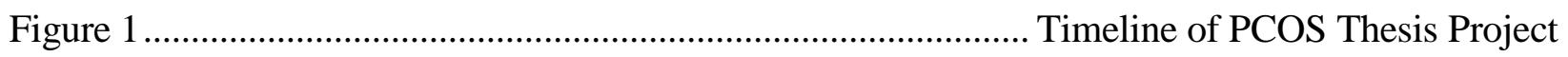

Figure 2 ....................................................... USA Regional Breakdown of Survey Respondents

Figure 3 ..................................................... World Regional Breakdown of Survey Respondents

Figure 4 ............................. Breakdown of PCOS Diagnostic Criteria Used by Survey Responders

Figure 5 .................................................Self-Reported Ratings for the Responders' PCOS Facility

Figure 6.................................Patient Satisfaction Data as Self-Reported from Survey Responders 


\section{List of Tables}

Table 1 Diagnostic Criteria for PCOS

Table 2 .Outline of Focus Group Questions

Table 3 Demographic Characteristics of Provider Specialty

Table 4 Breakdown of Physician Responders' Area of Expertise

Table 5 .Demographic Characteristics of Survey Respondents

Table 6 Breakdown of Specialty Providers Involved in Multidisciplinary PCOS Clinics

Table 7 BMI Category of Patients Treated for PCOS by Survey Responders

Table 8 Ideal Involvement of Specialties in Future Multidisciplinary PCOS Clinics 


\section{List of Abbreviations}

PCOS - Polycystic Ovarian Syndrome

$\mathrm{NIH}$ - National Institute of Health

ESHRE - European Society of Human Reproduction and Embryology

ASRM - American Society for Reproductive Medicine

AE - Androgen Excess Society

ROT - Rotterdam criteria

US - United States of America

BMI - Body Mass Index

HOMA - Homeostasis Model Assessment

ICD-9 - International Classification of Diseases, $9^{\text {th }}$ Revision

NASPAG - North American Society of Pediatric and Adolescent Gynecology

TSH - Thyroid Stimulation Hormone

OGTT - Oral Glucose Tolerance Test

F-G - Ferriman-Gallwey Score

UK - United Kingdom

IRB - Institutional Review Board

WVU - West Virginia University

SAHM - Society for Adolescent Health and Medicine

SART - Society of Assisted Reproductive Technology

ASRM - American Society of Reproductive Medicine

RD - Registered Dietitian

MD - Medical Doctor

NP - Nurse Practitioner

PA - Physician Assistant

LN - Licensed Nutritionist

CNS - Certified Nutrition Specialist

UK - United Kingdom

HCP - Health Care Providers 


\section{Chapter I: Introduction}

\section{i. Problem}

Polycystic ovarian syndrome (PCOS) is thought to be the most common endocrine disorder found in women. ${ }^{1,2}$ PCOS impacts women of all races and ethnicities who are of reproductive age. In unspecified populations the prevalence of PCOS has a reported incidence rate of $3-10 \% .^{3,4}$ PCOS is a syndrome that is seen only in women and is most often characterized by an imbalance of the sex hormones. ${ }^{1}$ Common symptoms include irregular menstrual cycles, ovarian cysts, and hirsutism. ${ }^{2}$ Features of the syndrome may also include infertility, insulin-resistance, impaired glucose tolerance (Type 2 Diabetes), dyslipidemia, and cardiovascular disease due to increased risk factors. ${ }^{1,5}$ The etiology of PCOS is not completely understood and there is no known cause, although a genetic component and lifestyle influences

have been identified. ${ }^{1,2,5}$ Due to the heterogeneous and multifactorial nature of PCOS symptoms there is a lack of a clear universal consensus regarding the definition and diagnostic criteria. ${ }^{1,6}$

Individuals with PCOS are in need of specialized, individualized, and focused care from a variety of health care providers who can work as a team to treat PCOS comprehensively. There is a large gap in the literature with very few studies documenting or assessing multidisciplinary PCOS treatment facilities. The limited research documenting the efficacy of multidisciplinary PCOS clinic have demonstrated increased weight loss, high patient satisfaction rates, and high retention rates. $^{7,8}$

The current literature lacks the perspectives of specific health care providers (HCP) on PCOS and enough supporting evidence on the benefits of dietitians for PCOS treatment. To our knowledge this is the first study that seeks to gain insight from a mix of HCP who frequently treat PCOS patients on the potential implications of multidisciplinary clinics and the challenges of involving dietitians in the care of PCOS. 


\section{ii. Hypothesis and Objectives}

Due to the complexity and heterogeneity of polycystic ovary syndrome, practitioners who work with patients with PCOS will describe the benefits of specialized, individualized, and multidisciplinary care. It is hypothesized that dietitians are not being utilization to their maximum involvement due to a variety of barriers.

In order to test the hypothesis the formative study's objectives were to:

1. To investigate the current trends in multidisciplinary treatment of PCOS across different provider types

2. To describe potential implications of future multidisciplinary PCOS clinics

3. To explore the role, importance and challenges for RDs in multidisciplinary PCOS treatment

\section{iii. Limitations}

Our survey sample did not allow for an associative analysis, to explore the potential association between different providers or type of treatment with various methods or outcomes. Also, the survey relied on self-reported data with no means of verification of credentials or experience. Several limitations were imposed on this study that accompany the nature of convenience sampling and focus groups. This study did seek the opinions of experts and it was not meant to be generalizable to the entire population of health care providers. It is possible that different experts would have different opinions in regards to PCOS but after reaching saturation it is unlikely the results would have been significantly affected. This purposive sample provided access to rich qualitative data that cannot be gathered though a broad-spread survey. 


\section{Chapter II: Review of the Literature}

\section{i. Clinical Presentation of PCOS}

PCOS is the most reproductive endocrine disorder in females. ${ }^{1,2}$ PCOS has a variety of phenotypes therefore is presents with a broad spectrum of clinical symptoms and risk factors. Insulin resistance is thought to play a central role in the etiology of PCOS and is present in 5090\% of women with PCOS (depending on diagnostic criteria used), which is significantly worse than age and BMI-matched control women. ${ }^{9}$ It has been estimated that $38-88 \%$ of women with PCOS are overweight or obese across the world, with an increased rate in the United States to mirror the higher obesity rates in the non-PCOS population. ${ }^{9}$ Insulin resistance does present in individuals with lean PCOS as well as overweight and obese women.

Another characteristic feature of PCOS in hyperandrogenism, which refers to elevated male-hormones (androgens), such as testosterone. Hyperandrogenemia can be diagnosed clinically through the presence of acne, hirsutism (unwanted hair growth around the face, chest, or trunk), or alopecia (male-pattern baldness or the thinning of hair). It can also be diagnosed biochemically through a blood test. In a large study of over 1,000 women with androgen excess, 659 presented with hirsutism and $78.4 \%$ of the hirsute women were diagnosed with PCOS under the 1990 NIH criteria. ${ }^{10}$

PCOS is often associated with infertility, which presents in an estimated $40 \%$ of women diagnosed with PCOS. ${ }^{11}$ The root of infertility in these women is likely from the menstrual disturbances, which is often presented as oligomenorrhea (with 85-90\% of women with PCOS), amenorrhea (presents in 30-40\% of women with PCOS), or abnormally long or erratic menstrual patterns. ${ }^{11}$ It is also important to mention that up to $30 \%$ women diagnosed with PCOS have normal menstrual cycles, ${ }^{11}$ which emphasizes the high degree of heterogeneity in this condition. 
Mental health outcomes are also of a concern when it comes to a multifaceted condition with dermatological symptoms, weight gain, fertility issues, as well as a variety of risk factors. Shakerardekani et al. conducted a cross sectional, multi-centric study in Iran on 100 women with PCOS and found that $45 \%$ presented with depression and $30 \%$ were considered for possible cases of other metal disorders. ${ }^{12}$ Another study by Coffey et al. in the UK found that PCOS had a negative impact on health related quality of life, even when compared with a variety of other health conditions with a relatively small sample size but while using a reliable and valid evaluation tool. $^{13}$

\section{ii. Geographical Prevalence of PCOS}

In the current literature there is a large disconnect between the prevalence of PCOS, geographical regions and race/ethnic factors. There are few studies that have examined specific subpopulations. These studies are commonly limited by small sample sizes, selection bias, and are not comparable with other studies' findings due to inconsistencies with the diagnostic criteria for PCOS. In order to fully understand the complexity and occurrence of PCOS, the prevalence needs to be assessed in the subpopulations. It is important for the field to reach the level of comprehension with PCOS to the extent that diabetes and metabolic syndrome established in

order to improve treatment. ${ }^{14,15}$ This review will determine the prevalence of polycystic ovarian syndrome based on geographical location and race/ethnicity. This will help to determine how much is understood regarding the risk and diagnosis of PCOS in specific regions of the world.

\section{Understanding the Prevalence of PCOS}

In order to begin to understand what is currently known about the prevalence in subgroup populations of PCOS the complexity and issues of the current diagnostic criteria must be understood. There are three different sets of diagnostic criteria that used in the field which have 
been set by National Institutes of Health's (NIH) international conference on PCOS in 1990, the European Society of Human Reproduction and Embryology and the American Society for Reproductive Medicine (ESHRE/ASRM) in 2003 (referred to as the Rotterdam criteria), and the Androgen Excess Society \& PCOS Society (AE-PCOS) in 2006. ${ }^{5,16-18}$ Each set of criteria has slightly different clinical, biological, and image-based findings to determine the presence or absence of PCOS. ${ }^{1}$ The 1990 NIH Criteria suggest that a patient has PCOS if she displays symptoms of oligoovulation and androgen excess (clinical or biochemical). ${ }^{5}$ The Rotterdam 2003 Criteria was developed in response to a need for broader diagnostic criteria. ${ }^{1}$ In order to be diagnosed with PCOS under the Rotterdam criteria (ROT) the individual must exhibit symptoms in two out of the three categories, which include oligo/anovulation, hyperandrogenism, and the presence of polycystic ovaries. ${ }^{5}$ The most recent criteria that was published by the AE-PCOS in 2006 tightened the criteria to include all three symptoms used in the Rotterdam criteria in an effort to give an evidence-based definition to PCOS. ${ }^{5,16}$ All three diagnostic criteria sets have specific exclusion criteria that differ. The diagnostic criteria is constantly evolving and is considered to be one of the most debated topics in the field of endocrinology ${ }^{16}$ making the prevalence of PCOS difficult to determine with consistency. It has been previously stated that because PCOS is a clinical syndrome, and there is no criteria that is fully sufficient for diagnosis. ${ }^{19}$

Table 1: Diagnostic Criteria for PCOS

\begin{tabular}{|l|l|l|}
\hline \multicolumn{2}{|c|}{ NIH 1990 } & \multicolumn{1}{c|}{ Rotterdam 2003 } \\
\hline $\begin{array}{l}\text { - Hyperandrogenism } \\
\text { Chronic Anovulation }\end{array}$ & $\begin{array}{l}\text { - Hyperandrogenism } \\
\text { - Oligo-and/or anovulation } \\
\text { - Polycystic ovaries } \\
---2 \text { of 3 criteria needed }\end{array}$ & $\begin{array}{l}\text { - Hyperandrogenism } \\
\text { - Ovarian dysfunction }\end{array}$ \\
\hline $\begin{array}{l}\text { First developed and most } \\
\text { commonly used criteria today }\end{array}$ & $\begin{array}{l}\text { Formulated to expand on NIH } \\
\text { diagnostic definition }\end{array}$ & $\begin{array}{l}\text { Formulated to provide an evidence- } \\
\text { based definition }\end{array}$ \\
\hline
\end{tabular}




\section{Effect of the Diagnostic Criteria on Prevalence}

Changes in the diagnostic criteria greatly affect the prevalence of PCOS. Prevalence rates have been reported as low as $1.6 \%$ using a combination of all three criteria ${ }^{1}$ and as high as $18 \%$

2 in similar Caucasian populations using the Rotterdam criteria. ${ }^{20,21}$ A statistical report by Futterweit, estimated that $50-75 \%$ of women with PCOS are unaware that they even have this syndrome. ${ }^{22}$ A retrospective cohort study by Amato et al. ${ }^{16}$ assessed a group of 204 agematched women who were suspected to have PCOS to determine the difference in prevalence based on the diagnostic criteria. This study found that the prevalence of PCOS in the identified population to be $51 \%$ according to NIH, $83 \%$ with ROT, $70.6 \%$ with AE-PCOS, and only $49 \%$ to fit the PCOS diagnosis under all three categorical descriptions. ${ }^{16}$ These findings all showed a difference in the prevalence as well as the frequency and severity of symptoms. In a cohort study $^{6}$ using a large anovulation-screening database all cases were assessed under the Rotterdam criteria and then redefined and diagnosed under the $\mathrm{NIH}$ criteria to determine the prevalence of PCOS according to the two different definitions. When the subjects were diagnosed according to the Rotterdam criteria there was a 1.5 times larger group that was diagnosed than when the same subjects were diagnosed using the NIH criteria. Under the Rotterdam criteria there was a greater frequency of obesity, insulin sensitivity, and the diagnosis of PCOS itself. ${ }^{6}$ This study used appropriate groups and used the same subjects to assess an accurate depiction of the differences that can occur between the criteria. Another study ${ }^{2}$ found that the Rotterdam and AES prevalence estimates were nearly twice that of the NIH criteria when classified on the same participants. The lack of consistency and clarity between the diagnosis criteria affects the comparability and the standardization of all clinical treatments and research findings dealing with PCOS. 
There is a limited amount of literature on previous research regarding the prevalence of PCOS dependent on a geographical location, specific race or ethnicity, or how the prevalence of PCOS is related to the occurrence of additional health disparities. Previous studies are commonly limited by a small sample size, fewer than 400 , conducted at only one facility, and are not fully comparable due to the lack of consistency in the use of diagnostic criteria. ${ }^{1}$ It has been stated that there is a significant difference seen in the symptoms presented across geographical locations and between differing race/ethnic groups. ${ }^{23}$

\section{Prevalence of PCOS Across the US}

To our knowledge, Okoroh et al. ${ }^{1}$ is the only study to assess and compare regional prevalence of PCOS and its various phenotypes across the United States (US) and the first to use all available criteria to estimate the prevalence in the US. This is also believed to be the one of the largest prevalence studies done on a geographically diverse population within the United States. This research showed a higher prevalence of PCOS concentrated in the southern US than anywhere else in the US. This study showed that following the South, in order of decreasing prevalence, was the North Central, West, and then the North East with the lowest prevalence. ${ }^{1}$ This was a large scale study that analyzed a commercial database containing claim reports that were collected from 2003-2008 looking at over 12 million privately insured women aged 18-45 from geographically diverse states. Only $1.6 \%$ of women met at least one diagnostic criteria for PCOS. ${ }^{1}$ This prevalence may be a low estimate since this a retrospective study only had access to medical charts previously completed and did not see the patients directly for an extensive clinical exam. There is also the possibility of the information on the charts being improperly coded leading to missed diagnosis. Since it is not uncommon for PCOS to go undiagnosed, it is extremely plausible that this article underestimates the prevalence of PCOS significantly. 


\section{Prevalence of PCOS in Caucasians Across the World}

Various studies have assessed Caucasians in the US, Spain, Australia, and Greece to determine whether or not Caucasian populations in other countries showed similar prevalence rates across the world.

A previous study, by Asuncion et al., ${ }^{24}$ prospectively estimated the prevalence of PCOS using a design similar to Knochenhauer et al. ${ }^{3}$ that selected women in an unbiased manner by using data from 154 consecutive Caucasian blood donors at a hospital in Madrid, Spain. Using the NIH diagnostic criteria, the study found an incidence rate for PCOS of $6.5 \% .^{24}$ One limitation of this study was that is was a small study and although the selection was not biased, it was not completely randomized and is unlikely to be representative of the population in that area.

A large retrospective birth cohort was designed by March et al. ${ }^{2}$ to create a representative estimate of the prevalence of PCOS in those born in Adelaide, Australia. 728 female reproductive age subjects were assessed that were all born at a single maternity hospital and could be located, interviewed, and clinically examined. This study took into consideration the lack of consistency between the diagnostic criteria and assessed the patients according to each criteria to determine prevalence rates specific to the criteria. The study determined a prevalence of $8.7 \pm 2.0 \%, 11.9 \pm 2.4 \%, 10.2 \pm 2.2 \%$ according to NIH, Rotterdam, and the AES criteria respectively. These numbers increased to $17.8 \pm 2.8 \%$ under the Rotterdam criteria and $12.0 \pm 2.4 \%$ according the AES criteria when imputed data was included for those women who did not have an ultrasound. ${ }^{2}$ The main strengths of this study were that is the largest and only community-based study that looked at the prevalence of PCOS in a nearly homogeneous Caucasian population. Although these incidence rates seem higher than those determined in the United States this population was primarily Caucasian and is said to be comparable to the US, ${ }^{25}$ 
in terms of obesity rates and waist circumference, the rates of Australians were still lower than in

the Americans. ${ }^{26-28}$ While the previous study looked primarily at Caucasian Australian women, which made up $94 \%$ of their participants, other studies have looked at Australian indigenous women and found very different results that are discussed below. ${ }^{29}$

\section{Prevalence of PCOS Across Different Races/Ethnicities}

Studies have suggested that the prevalence of PCOS may vary between different races and ethnicities. The following studies assessed the prevalence of PCOS looking at specific race/ethnicities in a single geographical area.

Due the wide-variety of ethnic groups of Asians it is expected that the variance or symptoms between individuals' different ethnicities will vary and this has been documented across multiple studies. A community-based, cross-sectional study assessed a random sample that was representative of the community of over 3,000 women between the ages of 15 and 39 in Sri Lanka. The study by Kumarapeli, et al., ${ }^{30}$ found a prevalence rate of $6.3 \%$ (95\% CI: 5.9$6.8 \%$ ) based on the Rotterdam criteria. This study used a survey to first narrow down the probable cases and controls and then performed a clinical examination to further deduce probable cases and then used ultrasound tests to confirm the identified PCOS cases. Over 90 percent of women self-reported symptoms of oligo/amenorrhea and/or hirsutism were confirmed to have PCOS according to Rotterdam criteria. ${ }^{30}$ This method showed that a simple questionnaire-based survey may be an effective and simple tool that could be used for PCOS screening in South Asia and even other areas of the world. In this study only $0.65 \%$ of those with PCOS had been previously diagnosed. It is suspected that there would be an increased prevalence of PCOS in Sri Lankan women when compared with Caucasians due to the known link between type 2 Diabetes Mellitus and the high prevalence of diabetes in Sri Lanka. ${ }^{30,31}$ Due 
to differing diagnostic criteria used in similar studies in the US it is difficult to compare the results directly to determine the similarity. These findings were consistent with those documented in Southern Europe. ${ }^{30}$ It has been reported that the prevalence of PCOS is considered to be higher in South Asians than in Caucasians residing in the United Kingdom. ${ }^{32}$ Fifty-two percent of all Asian women who reside in the Indian subcontinent have been found to present with polycystic ovaries, which is considered to the highest reported prevalence. ${ }^{33}$ The estimated prevalence of women with polycystic ovaries in the US is approximately $21 \%$ of a select population. ${ }^{34}$ Although Japan has lower rates of obesity and hirsutism, the Japanese still have comparable rates of androgen excess and insulin resistance to the US and Italy. ${ }^{23}$ No known studies have been published describing the prevalence of PCOS in Japan or Italy in order to compare the populations further.

According to Moran, ${ }^{35}$ a prevalence of $6.0 \%$ (95\% CI: 1.9-10.1\%), as diagnosed by the NIH criteria, or $6.6 \%$ (95\% CI: $2.3-10.9 \%$ ) under the Rotterdam criteria was found in a homogenous group of Mexican women residing in Mexico City. This study could have been limited by a small sample size of 150 women who all joined the study voluntarily. Strengths of this study include the use of two diagnostic criteria sets and the fact that they were all assessed clinically, biochemically and by a pelvic ultrasound. ${ }^{35}$

According to Goodarzi et al., ${ }^{36}$ a significantly higher PCOS prevalence has been documented in Mexican-American women living in Los Angeles that is approaching 13\%. 4,35,36 This study prevalence could be impacted by a confounding factor from bias stemming from their selection of individuals who all had a family history of coronary artery disease. There was no clinical evaluation with the subjects because this study relied solely on self-reported data via a 
questionnaire, which could significantly skew their results. Unlike most studies this study failed to remove patients with hyperandrogenism connected with a related disorder.

The differences between these studies focused on Mexican women could imply that there is a difference in lifestyle from those residing in Mexico vs. United States. If the experimental designs were similar in methodology and better controlled then this assumption could be stated with more confidence. It is plausible that there is a higher prevalence of PCOS in MexicanAmericans because in a study comparing Caucasian women and Mexican-American women with PCOS found that they have been found to have a higher age-specific prevalence of insulin resistance and a higher body mass index (BMI) when compared to non-Hispanic Whites. The study used a smaller sample size of 83 participants but consistently observed significantly higher mean values for BMI, fasting insulin, and homeostasis model assessment (HOMA) in MexicanAmerican women compared to the Caucasian women. ${ }^{4}$ One of the most prominent features of PCOS is insulin resistance, which is found in $50-70 \%$ of individuals with PCOS. ${ }^{36}$

Despite the correlation between a higher prevalence of PCOS and a higher Black population in the Southern US, Knonchenhauer et al., ${ }^{3}$ found that there are no significant racial differences between Whites and Blacks living in Alabama with a prevalence of $4.7 \%$ and 3.4\%, respectively. ${ }^{3}$ This study had a sample size of 369 women in the Southeastern United States that were between the ages of 18-45 who were examined as part of a pre-employment physical. The subjects were assessed for PCOS according to the NIH guidelines. This study was detailed and avoided bias in the selection of their participants although, due to the chosen diagnostic criteria it did not include the polycystic ovarian morphology or ultrasound as part of the examination.

Other studies by Azziz et al., ${ }^{20}$ have examined this hypothesis using the same database and the same criteria. These studies confirmed the finding of no significant difference, showing a 
prevalence of $8.0 \%$ for Black women and $4.8 \%$ for White women in Alabama, potentially due to a small sample size $(\mathrm{n}=400) .^{20}$

Davis et al. reported results that are suggestive of a preliminary indication that indigenous Australian women could have a prevalence as high as $26 \%$. It is important to note that this study is limited by its small sample size $(\mathrm{N}=38)$ and a PCOS diagnosis based off of the presence of oligomenorrhea and hirsutism and/or hyperandrogenemia. The measures collected from the participants included hirsutism (from facial scoring only), BMI and waist circumference, insulin and glucose levels, and hormone analysis to test total testosterone and sex hormone binding globulin (SHBG), which were used to calculate a free androgen index. ${ }^{29}$ It was expected that the prevalence found in the indigenous people would vary from the rest of the population due to their rapid change from a hunter-gatherer way of life to a sedentary life-style with a high-fat and nutritionally poor diet. This population has especially high rates of hirsutism, central obesity, and type 2 diabetes compared to Caucasians. ${ }^{29}$ These symptoms are symptoms of PCOS and the increased prevalence may be attributed to PCOS or increase their risk for developing PCOS.

A cross-sectional study that assessed 192 women between the ages of 17 and 45 who were living on the Greek island of Lesbos determined the prevalence of PCOS, according to the NIH criteria to be $6.77 \% .{ }^{37}$ This study recruited participants via a convenience sample by accepting those who responded to their offer of a free medical examination by an endocrinologist. This method introduced bias and may have altered the results by attracting more individuals who think they need to see a doctor than those who consider themselves healthy. Regardless of the potential bias, this value parallels the typical prevalence rate in the United States. $^{3}$ 


\section{PCOS Prevalence Conclusion}

Based on the NIH diagnostic criteria, there is a similar prevalence of PCOS between 69\% documented across the United States, the United Kingdom, Spain, Greece, Australia, and Mexico. ${ }^{38}$ This information suggests that there are no racial or ethnic influences on the prevalence of PCOS. Due to the lack of comparability amongst the studies, biased group selections, and small sample sizes it is recommended that further research be conducted before this generalized statement is accepted. There are multiple hypothesized reasons for the lack of understanding of the risk and diagnosis of PCOS and one main reason could be the conflicting diagnostic criteria. The different components of the diagnostic criteria cause alterations in the prevalence across the NIH 1990 Criteria, Rotterdam 2003 Criteria, and AE-PCOS 2006 Criteria.

${ }^{5}$ National prevalence rates have been reported as low as $1.6 \%$ using a combination of all three criteria ${ }^{1}$ and as high as $6.6 \%$ using $1990 \mathrm{NIH}$ criteria in similar American populations. ${ }^{20}$ One strength that was noted between all studies was that they all observed a similar age group to depict women of reproductive age. In particular, most of the studies looked at the age group between 18-45. 1,3,20,24,37 There is limited literature that exists but there has been similar prevalence rates between Whites of European decent, African-American, and Mexican women noted. ${ }^{38}$ Due to the inconsistency between diagnostic criteria and recruiting methods it is unlikely that all studies in this review are comparable enough to infer conclusive differences upon. The existing data is not conclusive enough to determine whether or not there is any significant differences in the prevalence of PCOS across geographical location, racial or ethnic groups.

Future research is needed to determine a better diagnostic criteria and ways to improve diagnosis so that less individuals with PCOS are undiagnosed. These will be the first steps to 
determining a more accurate prevalence, which can then be assessed according to subpopulations to achieve a better understanding of this multifaceted syndrome. This topic is in need of large-scale, random, populational studies across the world that look at the prevalence of PCOS according to the all of the established diagnostic criteria is specific sub-populations that can be repeated with many different sub-groups.

\section{iii. The Current Description and Future Need for Polycystic Ovary Syndrome Clinics}

Polycystic ovarian syndrome (PCOS) is thought to be the most common endocrine disorder found in women. ${ }^{1,2}$ PCOS impacts women of all races and ethnicities who are of childbearing age. PCOS is associated with a significant increase in risk factors such as cardiovascular disease, type 2 diabetes, and infertility. ${ }^{5}$ The etiology of PCOS is not completely understood, although a genetic component has been identified. ${ }^{5}$ Given the heterogeneity of its symptoms and constantly evolving (and debated) diagnostic criteria, the prevalence PCOS can be difficult to pinpoint. The prevalence of PCOS is commonly thought to vary between 5-10\% depending on diagnostic criteria and sample population. ${ }^{1,2,5}$ It has been previously stated that because PCOS is a clinical syndrome, and there is no criteria that is fully sufficient for diagnosis

19 The main goal of this review is to determine the need for multidisciplinary PCOS clinics based on their contribution to treatment outcomes and assess the current literature on existing multidisciplinary PCOS clinics.

\section{Significance of the Problem}

PCOS is a multifaceted and complex syndrome that requires care from multiple providers to fully treat the full spectrum of PCOS. It can be difficult to treat due to its heterogeneity between patients, which therefore requires specialized, individualized and focused care. PCOS is most often diagnosed in adolescents struggling with menstrual issues or women with infertility; 
combine those issues with hirsutism, high insulin levels, weight-gain, and acne ${ }^{1}$ and this leads to a vulnerable and frustrated population. There are many aspects involved in evaluating and treating PCOS patients, including: regulating the menstrual cycle, addressing metabolic abnormalities, normalizing nutrition (i.e. weight loss if overweight), psychological treatment for poor self-image, depression, and anxiety, and addressing concerns such as infertility and increased risk of comorbidities. ${ }^{7,39}$ A multidisciplinary treatment approach to PCOS is thought to be an effective strategy that will enable the coordination of care and also serve as an innovative platform for research on the full spectrum of PCOS. ${ }^{8}$

Another reason to support the need for multidisciplinary PCOS clinics is the high rate of PCOS patients who remain undiagnosed when only seeing one specialist. In a study by BroderFingert et al. ${ }^{40}$ data was collected on an inner-city clinic by a retrospective chart review in a hospital based pediatric clinic in New York City. Data was analyzed from 60 female patients between the age of 13 and 19 with a primary ICD-9 diagnosis of ovarian dysfunction, menstrual irregularity, or hirsutism who were selected randomly. The primary outcome of this study looked at the rates of assessment for the diagnostic criteria of PCOS and selected co-morbidities. Only $25 \%$ of the patients in the study with suspected PCOS (according to any of the three common diagnostic criterias) were evaluated for PCOS. ${ }^{40}$ Only 2 patients $(3.33 \%)$ were evaluated for common co-morbidities associated with PCOS. ${ }^{40}$ A full evaluation for PCOS at this clinic included: menstrual irregularities, hirsutism, ovarian ultrasound, fasting glucose, fasting insulin, lipid profile (HDL, LDL and triglycerides), testosterone, and FSH to LH ratio. Twenty-eight out of 60 patients presented with menstrual irregularity in addition to one of the following signs: obesity, hirsutism, and/or acne. ${ }^{40}$ Of those 28 patients only 15 (54\%) were evaluated for PCOS, according to the Rotterdam Criteria, and only $7 \%$ were evaluated for co-morbidities. ${ }^{40}$ As 
demonstrated by the lack of PCOS evaluation in patients with one or more symptoms demonstrates that PCOS is under-evaluated and under-diagnosed in this pediatric population and most likely throughout the US. This study's strengths included their exhaustive evaluation measures to diagnosis PCOS and the fact that this the first study to assess the rate of undiagnosed PCOS cases in an inner-city population. Due to the reliance on a retrospective chart review there is a possibility of inadequate or incomplete medical chart reporting, which could alter the rate of existing diagnosis.

Sivayoganathan et al. showed that the percent of patients that go undiagnosed with PCOS might vary greatly depending on the type of clinic. The data for this study was collected on 70 women using a prospective cross-sectional observational study at four different clinics at Leeds General Infirmary in the United Kingdom. Participants were all assessed for PCOS by a full endocrine and metabolic profile as well as an ultrasound. In this study, $65 \%$ attending the dermatology clinic, 38\% attending the endocrinology clinic, 25\% attending the gynecology clinic, and $15 \%$ attending the fertility clinic were confirmed by this study to have PCOS without a pre-existing evaluation. ${ }^{41}$ The difference between the rates of existing diagnosis was shown to be significant $(\mathrm{p}=0.0088) .{ }^{41}$ Even though all participants experienced menstrual problems there was a significant difference $(\mathrm{p}<0.0234)$ with menstrual patterns and the frequency distribution of related symptoms between types of clinics. ${ }^{41}$ This study also found that four out of six participants who were found to have diabetes were diagnosed through this study, indicating possible missed diagnosis of a medically important disorder. ${ }^{41}$ Most women were receiving an oral-glucose test for the first time. This was the first study to compare four different clinics that often treat the symptoms of PCOS, and it also used an exhaustive evaluation for PCOS. This 
study may have been limited by a relatively small number of participants but significant results were still found.

\section{Efficacy of Multidisciplinary PCOS Clinics}

Very few studies have been done to assess multidisciplinary treatment for PCOS. It is important to understand the clinical variability that is seen between treatment providers, success in existing clinics, and additional research promoting the use of a multidisciplinary treatment team. A cross-sectional study to assess practice heterogeneity by Bonny et al. conducted an anonymous Internet survey that yielded 127 responses from North American Society of Pediatric Adolescent Gynecology (NASPAG) members. When the respondents were asked to select their expertise (more than one could be selected) $64 \%$ selected gynecology, $43 \%$ selected adolescent medicine, $13 \%$ selected reproductive endocrinology, $6 \%$ selected general pediatrics, and $4 \%$ selected endocrinology. ${ }^{42}$ The most common first-line treatment therapies included the prescription of oral contraceptives followed by diet modification and exercise with $98 \%$ and $90 \%$ of respondents, respectively. ${ }^{42}$ While $65 \%$ of clinicians would not make a diagnosis for PCOS within the first 2 years after menarche, $35 \%$ of respondents would. ${ }^{42}$ Only $60 \%$ of respondents noted that they look at blood glucose levels at an initial PCOS evaluation. ${ }^{42}$ Thyroid stimulating hormone (TSH) was the most common test completed at an initial PCOS evaluation with just below $90 \% .^{42}$ There was a high degree of variability in the hormonal and metabolic evaluations. The high degree of variability in evaluation is evidenced by the fact that there was not one test used to diagnose PCOS that was ordered by all clinicians. Even though this survey targeted the experts in the field who have an interest in PCOS, there was a considerable amount of heterogeneity within the first diagnostic testing that was normally completed. This study was 
limited due to it being a pilot study that was designed to assess descriptive statistics only, but it was the first study to address the input across a variety of providers on the evaluation of PCOS.

Currently, there are only two multidisciplinary PCOS treatment facilities that have published research regarding the outcomes of their clinics. The first multidisciplinary clinic to examine the benefits of such treatment is the one of the most well-known multidisciplinary PCOS clinics in the United States, which is at the American Family Children's Hospital in Madison, Wisconsin. This clinic has been in existence since 2005. This clinic's team consists of two pediatric endocrinologists, a reproductive endocrinologist, an endocrine nurse, a health psychologist, a dietitian, and a pediatric gynecologist. ${ }^{7,39}$ The following two studies were based off of data collected during the first 33 months of operation (March 2005 - December 2008) of this clinic. The goal of this study was to characterize patients referred to the adolescent PCOS clinic by conducting a chart review of all patients $(n=70)$ seen in the first 33 months. Bekx et al. collected data on initial presentation, age, body mass index (BMI), menstrual pattern, features of androgen excess, insulin resistance, and dyslipidemia. The average age of the patients at the time of referral was 16.2 years old but ranged from 11 to 22 years old. ${ }^{39}$ Eighty-four percent of patients had a BMI above the $85^{\text {th }}$ percentile and $70 \%$ had a BMI greater than the $95^{\text {th }}$ percentile. 39 They saw a great amount of variance in menstrual patterns and that ranged from primary amenorrhea to regular cycles. Over $50 \%$ of patients showed signs of hirsutism. ${ }^{39}$ Only three cases of type 2 diabetes were confirmed, with two being pre-diagnosed and one diagnosis given through an oral glucose tolerance test (OGTT). ${ }^{39}$ More than $50 \%$ of patients were thought to have insulin resistance that was demonstrated by elevated fasting insulin levels or a fasting glucose-to-insulin ratio of less than 4.5. ${ }^{39}$ Twenty-four percent of patients had elevated fasting triglyceride levels above $150 \mathrm{mg} / \mathrm{dL}$, and $54 \%$ had low HDL levels below $50 \mathrm{mg} / \mathrm{dL} .{ }^{39}$ Due to 
the retrospective design of the chart review for data collection, limitations may result from coding discrepancies. Also, because the patients were all at different stages of evaluation and treatment it could cause a variance or skew in data collected. This study did not use confirmatory testing to ensure the findings of various diagnostic measure due to the lack of financial justification. Missing data was a slight limitation because not all labs that were ordered were actually collected, although this is consistent with realistic expectations in clinical settings.

Geier et al. focused on assessing the providers seen by the patients, weight loss and retention rate at the same clinic. The data for this study was collected by a retrospective chart review that evaluated 140 adolescent females who had been seen at the PCOS Clinic between March of 2005 and December of 2008. The average age of patients at their initial visit to the clinic is $15.9 .^{7}$ The procedure of this PCOS clinic is to have all patients see each of the five providers at the initial visit. Only $41 \%$ saw all five providers during the initial visit. All patients at this clinic saw a pediatric endocrinologist and the endocrine nurse at their first visit and an additional, $60.9 \%$ see a health psychologist, $75.5 \%$ see a dietitian, and $70.9 \%$ see a gynecologist. ${ }^{7}$ Geier et al. found that nearly $70 \%$ of patients succeeded in short-term weight stabilization and $57 \%$ established weight loss. ${ }^{7}$ These patients had an average initial BMI of $34.7\left(\mathrm{~kg} / \mathrm{m}^{2}\right)$ and $76 \%$ had an initial BMI greater than the $95^{\text {th }}$ percentile. ${ }^{7}$ This study also found that $71 \%$ of patients returned for a follow-up visit with an average time of 4.5 months between visits. ${ }^{7}$ This high of a retention rate signifies that patients are generally satisfied with the treatment they are receiving. These high rates of success may be at least partially attributed to self-selection to seek weight-loss treatment indicating a pre-existing motivation to lose weight. These results might not be as pronounced in a PCOS population who have not yet reached that stage of change or in an adult population. 
The second clinic that has assessed the outcomes of multidisciplinary PCOS treatment is the Royal Berkshire Hospital in Reading, Berkshire, United Kingdom. The first study by Ghosh et al. was a two-year audit that was conducted on patients attending the multidisciplinary PCOS clinic from its opening in 2002 until 2004. They analyzed baseline characteristics, adequacy of investigations, efficacy of treatment, and patient satisfaction. The main complaints of the 127 women who attended this clinic were weight gain, hirsutisim and oligomenorrhoea. ${ }^{8}$ These women had a median age of 30 years and a median BMI of $32\left(\mathrm{~kg} / \mathrm{m}^{2}\right){ }^{8}$ The majority $(55 \%)$ of their patients were offered Metformin (to suppress glucose production in the liver) for treatment but 12 (17\%) discontinued use because of undesirable side effects. ${ }^{8}$ Patients using Metformin compared to those who were not did see a significantly greater amount of weight loss $(\mathrm{p}<0.0001)$ with a median loss of $8 \mathrm{~kg} .{ }^{8}$ Fifty percent of Metformin users with hirsutism saw an improvement in Ferriman Gallwey (F-G) score but it was less effective in those with very high initial F-G scores. ${ }^{8}$ Metformin also significantly improved menstrual cyclicity from a median of 20 weeks to five weeks $(\mathrm{p}<0.001) .{ }^{8}$ Eight of the 17 women taking Metformin and attempting to get pregnant had successful pregnancies in the two year time audit. ${ }^{8}$ Greater improvements to hirsutism levels were seen with the 23 women using Spironolactone (a diuretic that also reduces androgen levels). The median F-G score fell from 19 to 11 over an eight-month time frame. ${ }^{8}$ It is thought that there could be a higher patient satisfaction related to multidisciplinary clinics than with individual health care professionals. A study conducted at the Royal Berkshire Hospital in the UK showed that 42 out of 43 patients who completed a patient satisfaction survey reported that a multidisciplinary, dedicated PCOS clinic was "useful and that they were very happy with the results." 43 The patient satisfaction survey should be administered to a greater amount of 
patients to more adequately represent that clinic population as well as be more specific with the questions and response options for a better picture.

In 2007, Eldridge et al. ${ }^{44}$ conducted an audit on a nurse led PCOS weight management clinic at the Royal Berkshire Hospital in the United Kingdom. -his clinic had participants meeting once a month for individual appointments. The clinic focused on physical activity levels, use of food diaries, medications, changes in menstruation, and weight loss data. They found that there were 61 women who had attended clinic for at least two months. They had a mean starting BMI of 37.8. Out of the 37 women who attended for at least three months, 32 had lost weight with a mean loss of $2.88 \mathrm{~kg}$. Five of those 32 women lost $5 \%$ of their total body weight, two lost $7.5 \%$, and three participants lost $10 \%$. The average weight loss increased to $4.94 \mathrm{~kg}$ when women had attended for at least six months. Of the women who lost at least $5 \%$ of their body weight, $71 \%$ had kept a food diary, $86 \%$ had increased their exercise levels, $86 \%$ took Metformin, and $7 \%$ took Orlistat. ${ }^{44}$ Since participation was voluntary, these individuals were motivated enough to enroll and attend, which could increase their results.

The Androgen Excess PCOS Society (AE-PCOS) supports the emphasis of weight loss as part of lifestyle intervention for the primary treatment option for overweight and obese women with PCOS due to the strong association between obesity, abdominal obesity, insulin resistance and features of PCOS. ${ }^{45}$ Studies have shown than even a modest amount of weight loss, accounting to $5 \%$ of ones body weight, can reduce the severity of the symptoms for PCOS. ${ }^{45} \mathrm{~A}$ review by Moran et al., was conducted to determine the most effective way for women to lose weight. It was determined that achieving weight loss or even maintaining ones weight by preventing weight gain is best done with assistance from a multidisciplinary team. ${ }^{46}$ This multidisciplinary team should focus on lifestyle management that includes dietary, exercise, and 
behavioral therapy. ${ }^{46}$ Behavioral therapy should focus on psychosocial stress while openly discussing the practical and physiological barriers associated with weight management or weight loss.

Another review article by Moran et al. sought to compare the effect of different diet compositions on a variety of outcomes related to PCOS using the findings of six articles. There were only slight differences between the diets that have been tested with PCOS patients. They saw a slightly greater weight loss with a monounsaturated fat-enriched diet; improved menstrual regularity for a low-glycemic index diet; increased free androgen index diet; improved quality of life for a low-glycemic index diet; and improved depression and self-esteem for a high-protein diet. ${ }^{47}$ The findings of this compilation of research were inconclusive to support significant differences between any of the diet examined. The findings were conclusive to say that any diet aimed at reducing weight could lead to clinical benefits to those with PCOS. This indicates that the weight loss is more important than dietary composition when it comes to PCOS symptom management.

\section{Multidisciplinary PCOS Clinic Conclusions}

There is limited evidence directly related to multidisciplinary PCOS clinics and the efficacy of their treatment. It is well accepted that PCOS is multifaceted and has a high degree of heterogeneity among individuals with the syndrome. When treating a patient with PCOS it is important to focus on treating the patient's initial needs while decreasing the risk of long-term risk factors. Symptoms may be better treated if the patient is treated by a variety of specialists all working together. When individuals are exposed to multiple providers it is less likely that a PCOS diagnosis will be missed. It makes sense that the sooner PCOS is identified and treatment is initiated the quality of life of the patient and expression of the syndrome will improve. The 
perceived benefits of multidisciplinary clinics globally include improved patient satisfaction, greater weight loss, improved body image, and better management of PCOS from a holistic standpoint.

Further research is needed to assess additional existing multidisciplinary clinics to determine patient satisfaction and treatment prognosis compared to those seeking treatment from only one provider. More research is also warranted to gain a better understanding on evidence based guidelines for treatment of PCOS, especially when it comes to dietary recommendation.

\section{iv. Lifestyle Interventions and the Role of Registered Dietitians in the Treatment of PCOS}

\section{Lifestyle Interventions in the Treatment of PCOS}

It is important to realize that PCOS is a chronic disease with no known cure. PCOS typically requires a broad spectrum of treatment including pharmacology and lifestyle interventions to best manage the symptoms and disease risk associated with PCOS. Lifestyle interventions include the combination of dietary changes, increased physical activity, stress management and cessation of smoking. A general consensus in the current literature supports the use of lifestyle intervention as the first-line treatment for patients with PCOS, especially those who are overweight and obese. ${ }^{48}$ Looking beyond that broad statement, there is very limited evidence for specific dietary guidelines for the treatment of PCOS. The benefits of weight loss are prevalent in the literature but there is not enough data to support one optimal PCOS diet.

Insulin resistance is found in 50-70\% of women with PCOS, regardless of weight, therefore improving insulin resistance is a large focus of dietary and lifestyle interventions. ${ }^{49,50}$ Even modest weight loss, of 5\% of body weight, in overweight women with PCOS has been 
shown to improve a variety of reproductive, metabolic, and psychological features. ${ }^{48}$ Although the majority of research focuses on weight loss and the importance of dietary and lifestyle intervention in overweight women with PCOS, there are a small number of findings that support the importance of lifestyle management, including diet and exercise, that extend beyond weight loss to improve hypertension, insulin resistance, and elevated blood glucose levels. ${ }^{51-53}$

Various studies have looked at a variety of macronutrient ratios, low carbohydrate, high protein, altering fatty acid intake, and a low glycemic index (GI) or glycemic load but there is inadequate evidence to support the findings of an optimal diet for women with PCOS. ${ }^{54}$ Despite the fact that this review found no conclusive evidence for an optimal diet, it was shown that weight loss improved the presentation of PCOS in almost all of the studies regardless of dietary composition. ${ }^{54}$ This evidence supports the statement that weight loss should be targeted in all overweight or obese women with a diagnosis of PCOS through caloric restriction accompanied by adequate nutritional intake and healthy food selections regardless of the specific diet composition.

Currently there are no known published dietary guidelines or consensus statements in the United States for the dietary management of women with PCOS that have been put forth by a reputable organization.

\section{The Role of Registered Dietitians in the Treatment of PCOS}

Very few studies have explored the role of dietitians in the treatment of PCOS or the added benefit they could potentially provide. One study investigated the general attitudes towards diet and exercise, the extent of the implementation of lifestyle interventions that these women were provided, and the general knowledge that women with PCOS have about their condition. ${ }^{55}$ This study took place in the United Kingdom (UK) and interviewed 53 pre- 
menopausal women with a confirmed diagnosis of PCOS. This study found that all subjects identified the importance for weight management for PCOS yet only nine out of 35 (26\%) overweight women had ever received a referral to a dietitian. The majority of these women reported receiving most of information from the Internet and their endocrinologist who they typically see twice a year. Even though lifestyle intervention is recommended as the first-line treatment for PCOS, only $31 \%$ of overweight subjects reported being on a weight-reducing diet (with only $23 \%$ have success on their current diet) and $63 \%$ reported exercising regularly. This study found that $80 \%$ of overweight subjects reported weight loss to be difficult and only $26 \%$ had been referred to a dietitian so they were receiving very minimal assistance. ${ }^{55}$ It is suggested that by providing these patients with access to specialized dietitians and more frequent appointments the success rate for weight loss and clinical outcomes would be increased greatly. A study by Geier et al. ${ }^{7}$, that was discussed previously, has shown that the patients who saw the most success with weight loss had met with a dietitian and a health psychologist. This study, which provided multidisciplinary treatment, saw successful weight loss in $57 \%$ of patients who returned for a follow-up visit (1.5-12 months after initial visit with a mean interval of 4.5 months) and $70 \%$ of patients had weight stabilization. These differences can likely be attributed to the incorporation of dietitians and health psychologist to provide the proper nutrition and motivational counseling.

One study assessed the specific diet and lifestyle advice that was provided by 105 dietitians in the UK who worked frequently with PCOS patients. ${ }^{56}$ This study found that about one-third of dietitians that worked with PCOS worked together with other healthcare professionals in a multidisciplinary team approach. All dietitians reported recommending physical activity. The most common dietary approach recommended by these dietitians was to 
reduce energy intake $(78 \%)$ or select lower glycemic index foods $(77 \%)$, often times in conjunction with each other. Another approach was the alteration in macronutrients with the most common change, reported by $49 \%$ of responders, was lowering fat intake, followed by reducing carbohydrates (16\%), and only $3 \%$ advocating an increase in protein. Other general dietary advice commonly consisted of increasing fiber and reducing saturated fat intake. It was reported that overweight and obese patients were seen more frequently by dietitians, with only $24 \%$ of RD reported seeing patients with lean PCOS regularly. When asked about the different advice given to lean women with PCOS, dietitians reported primarily focusing on reducing the GI $(n=24)$, healthy eating $(n=9)$, and increasing physical activity $(n=6)$. It is important to point out that only $10 \%$ of dietitians reported having any departmental policy for the dietary management of PCOS. When looking at the level of confidence that these dietitians have in treating PCOS they found that only $34 \%$ of responders reported feeling well informed of the literature. This article supports the need for evidence-based guidelines for the dietary management of PCOS because $64 \%$ believed the available information is insufficient.

The second aspect of this study looked at the dietary habits of 203 women through a survey and a food diary and looked at where they got their information. ${ }^{56}$ This study found that only $15 \%$ of the 203 women with PCOS who completed the survey had ever seen a dietitian and that number reduced to $3 \%$ that had seen a dietitian during more than two appointments. When assessing where these patients got their nutritional information $22 \%$ reported using books and $21 \%$ received nutritional advice from their physician. This study also pointed out differences in referral and utilization of dietary resources for overweight/obese PCOS patients compared with lean women with PCOS. Overweight/obese patients were more likely to receive dietary advice from a dietitian $(21 \%)$ and a doctor $(25 \%)$ than lean women (10\% and $17 \%$, respectively). These 
women with PCOS were then asked to rate the effect of diet or lifestyle modification and $74 \%$ stated an improvement in PCOS symptoms and 19\% reported an improvement in weight loss. The highest reported perception of greatest improvement in symptoms, which was mentioned by $84 \%$ of the women who were increasing their physician activity (which, was $48 \%$ of respondents). Of the $32 \%$ of women with PCOS following a low GI index diet, $67 \%$ stated that they felt it improved their symptoms. Of the $30 \%$ who reported taking dietary/herbal supplement, 56\% reported a beneficial effect. This evidence supports the conclusion that PCOS is an important condition and dietitians should be aware of the condition and push to increase awareness.

A dietitian and exercise physiologist, Monika Woolsey, MS, RD, believes that there are a variety of challenges for women with PCOS in regards to working with health professionals in regards to diagnosing and treating PCOS. ${ }^{57}$ According to Woolsey, physicians tend to dismiss patients and not see endocrine disorders as a valid explanation for weight issues. When PCOS is not treated properly, and diet is not addressed, then symptoms can worsen. In Woolsey's opinion, she has seen dietitians face challenges gaining the trust from patients, because they are afraid of being judged or because previous advice did not help. Woolsey feels that dietitians are often times intimidated by endocrinology because it is complex, but in order to fully assist clients dietitians need to understand hormones. Woolsey concluded by saying that she believes "by not being proficient in working with this syndrome, we are not as effective as we could be in the fields of weight management, endocrinology, mental health, cardiology, bariatric and disordered eating."

There are no published guidelines on the nutritional management of PCOS by a recognized and reputable organization that currently exist. The closest thing is the American 
Dietetic Association's (ADA) position paper on weight management for adults in $2009^{58}$ but it is not specific to PCOS. This paper does highlight the importance for registered dietitians to remain skilled in aspects of weight management, individualized weight loss interventions, and the long-term sustainability of weight loss as part of their professional responsibility. To date, no published studies have examined the role of dietitians in the US in the management of PCOS nor have studies assessed the barriers dietitians face to get more involved with PCOS.

RDs are the food and nutrition experts and should be on the front-line working alongside physicians and other practitioners to manage this complex syndrome. There is an unmet need for position statements and evidence-based guidelines for weight-loss and management in overweight and obese individuals with PCOS, and dietary guidelines for normal weight PCOS patients. 


\section{Chapter III: Methodology}

\section{Study Design}

This formative study was designed to assess and describe current trends and potential implications in multidisciplinary treatment across multiple providers and in particular, investigate the role, importance and challenges for involving dietitians in the treatment of PCOS. This was a two-phase study that involved a preliminary survey to reach a broad category of providers to assess the current trends in PCOS treatment and explore potential implications for future multidisciplinary clinics though qualitative and quantitative data. The second phase of the study was designed to obtain rich-qualitative data that was more narrowly focused on the utilization, importance and challenges for involving dietitians in the treatment of PCOS. As Morse and Field described, qualitative research is meant to discover meaning and insight and not to measure the distribution of attitudes across an entire population, therefore randomization and representativeness is not a concern. $^{59}$

\section{Sample}

Phase one was a cross-sectional, anonymous, Internet survey. Phase two was a descriptive study that relied on a purposive, non-probability sample that was selected based upon theoretical sampling. According to Battaglia, the objective of obtaining a purposive sample is to logically assume the sample is representative of the population by applying expert knowledge of the population to select a sample that represents a cross-section of the given population. ${ }^{60}$ It is not the intent of this study to generalize the findings to the entire population of health care providers, but to gain feedback from the leaders in the field of PCOS on the impact and barriers regarding nutritional interventions and multidisciplinary PCOS treatment. Theoretical sampling is used to strategically select the best participants based on their ability to provide the most information-rich data. ${ }^{61}$ Morse and Field describe this as selecting a sample based on what has been learned from 
previous sources, ${ }^{59}$ which supported utilizing the data captured from the survey to effectively recruit for the focus groups. The sample size for neither phase was pre-determined and recruitment persisted throughout the duration of the data collection.

\section{PHASE I: Survey}

\section{Recruitment}

West Virginia University's Institutional Review Board (IRB) approved this study in April of 2013 (Appendix A). Practitioners who work with PCOS were invited to complete an Internet survey (Qualtrics, Provo, UT). An announcement was sent out asking for their participation in the survey to four list serves, various LinkedIn Groups (see Appendix C), and to individuals who were identified by their research or in the field or their involvement with existing PCOS treatment centers. Providers were encouraged to share the link with other providers so the response rate was unknown. The providers who chose to participate were instructed to click on the link in the email or posting that directed them to the online survey. A reminder email was sent out two weeks after the initial posting. The link opened up to the cover letter informing them of the implied consent and contact information of the research in case of any questions. The letter included information about the researchers, the study, and the approval status of the WVU IRB approval. The next page marked the beginning of the survey questionnaire, which was designed to take fifteen to twenty minutes to complete.

\section{Survey Instrument}

The survey was an internet-based survey through Qualtrics (Provo, UT), which consisted of 30 multiple-choice, multiple-response, and open-ended questions targeting information on their demographics, current treatment facility and approach, and perspectives about future multidisciplinary clinics. The open-ended questions allowed for more free-flowing feedback 
about their thoughts. This survey was designed based on current literature reviews and existing multidisciplinary clinic data and was formulated with the help of expert review. Professionals in the field including a physician, fertility specialist, dietitians, and a group of students piloted the survey for feedback. The final survey was released and left open for two months (May $15^{\text {th }}-$ July $\left.15^{\text {th }}, 2013\right)$. The survey can be viewed in Appendix E.

\section{Analysis}

Responses from the survey were downloaded from Qualtrics (Provo, UT) and cleaned from entry into SAS software (SAS 9.3, SAS Institute, Cary, NC) where the frequencies and averages were analyzed.

\section{PHASE II: Focus Group}

\section{Recruitment}

An addendum was submitted to the WVU IRB and approved this addition of a focus group to this study in January of 2014 (Appendix B). Theoretical sampling ${ }^{61}$ was used to select participants based on their potential to supply rich information. An invitation (Appendix F) was sent out to responders from the original survey who submitted their contact information and resided in the United States $(n=22)$ inviting them to participate in a focus group. If respondents left their phone number, they were also called for a verbal invitation $(n=13)$. Respondents were then asked for referrals to other professionals and the invitation was then extended $(n=4)$. There were an additional 12 email invitations sent out to health care professionals that were very specialized in PCOS and that were located via Internet search. As part of the grounded theory, theoretical sensitivity has been described by Glasser and Strauss as a way to 'describe the wisdom that

researchers bring to an inquiry. ${ }^{61,62}$ Once participants were identified a date and time sign up sheet was distributed. A total of nine providers engaged in the series of focus providing a participating 
rate of $24 \%$ of those contacted. Contact information and mailing addresses were collected for the sole purpose of mailing out the compensation after their participation in the focus group.

\section{Focus Group Data Collection}

Similar providers were placed together to promote group cohesiveness ${ }^{63,64}$ and compatibility; ${ }^{65,66}$ for example, physicians were paired with other physicians and dietitians were kept together as much as possible.

During the focus group, participants were asked to respond to a series of open-ended questions. In each of the focus groups, the following questions were asked:

Table 2: Outline of Focus Group Questions

\begin{tabular}{|l|}
\multicolumn{1}{|c|}{ Focus Group Question Outline } \\
\hline 1. Describe any nutritional interventions that you provide to your patients? \\
\hline 2. How is the dietary intervention and patient care communicated between providers? \\
\hline 3. When is dietary intervention warranted for a patient with PCOS? \\
\hline 4. How accessible are nutritional interventions for the majority of PCOS patients? \\
\hline 5. What are some of the challenges for getting dietitians more involved with PCOS? \\
\hline 6. Do you feel like providers know and understand the value of nutritional interventions for PCOS patients? \\
\hline 7. In your career, have you seen any shift in the awareness or interest of PCOS? \\
\hline
\end{tabular}

The script (Appendix G) included seven main questions to provide the general foundation for each focus group but still allowed for fluidity and room for additional comments. This template was used to ensure consistency for data collection but slight adjustments in prompts were made due the findings in the previous focus group to support to utilize constant comparison as part of the grounded theory. ${ }^{61,62}$ Holloway and Wheeler feel that this method increases the quantity of the data collected and helps to develop categories and theories with more saturation. ${ }^{67}$ Birks and Mills describe constant comparison as an inductive process that allows for the analysis to be grounded in 
the data and reconnects the data as the research develops the theory. ${ }^{68}$ The focus group format allowed the participants to engage in free-flowing conversation and promote the sharing of ideas, feelings, and experiences that went beyond the information that was captured by a simple questionnaire. The study participants were aware that there were no right or wrong answers and that confidentiality measures would be taken to remove their identifying information from any transcriptions. During the study, participants engaged with others via teleconference. All focus groups were audio-recorded so that they could be later transcribed.

\section{Analysis}

For each focus group there was three dedicated note takers and one facilitator who were kept consistent throughout the series. Braun and Clarke's method ${ }^{69}$ for thematic analysis in addition to the grounded theory ${ }^{62}$ was used to analyze the focus group data. In support of concurrent data collection and analysis, which is a fundamental component of the grounded theory, data was coded, reviewed, and discussed between each subsequent focus group. The focus groups were all audio-recorded and transcribed into word document that was typed by the researcher. The transcript was compared with each of the note-takers note to examine for discrepancies. The final transcription was analyzed question by question to identify themes and sub-themes. The transcripts were examined to determine how extensive the participants discussed topics. The transcriptions were reduced to exclude any unnecessary words to facilitate the identification of themes efficiently. Thematic analysis was used to sort through the reduced data. By using thematic analysis we are relying on the content analysis, which in a qualitative study focuses on intentionality and implications of the context. ${ }^{69}$ After the themes are identified and coded they are sorted and paired accordingly. Themes are identified with re-occurring context noted and theoretical saturation was research when new analysis only produced codes that fit into existing 
categories. Glasser defined theoretical saturation as part of the grounded theory as met once the properties and dimensions of the categories are fully explained. ${ }^{61}$

\section{Timeline}

The development of the survey tool used in this study took place from February 2013 April 2013. WVU's IRB approved the study in April of 2013 with an addendum for the focus group approved again in January 2014. Recruitment for the survey component of this study took place from April to June of 2014. The survey remained open from May $15^{\text {th }}$ to June $15^{\text {th }}, 2013$. Data analysis for the survey took place between July and November 2013. Survey participants who included their contact information in the survey were contacted and scheduled in January 2014 to recruit for the focus groups. Focus groups were held during the last week of January 2014. Data analysis for the focus groups took place in February 2014.

Figure 1: Timeline of PCOS Thesis Project

\section{PHASE 1: \\ Survey Tool \\ Development \\ February - \\ April 2013}

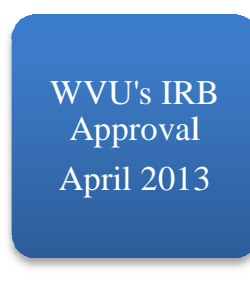

\section{PHASE 2: \\ Focus Group \\ Planning \\ November - \\ January}

WVU's IRB

Addendum

Approval

January 2014
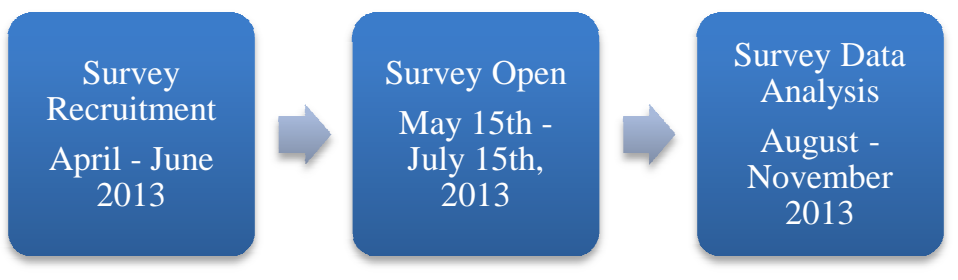

Focus Group

Recruitment

January 2014
Focus Group Data Analysis

February 2014 


\section{Chapter IV: Results}

\section{i. Survey Results}

\section{Demographics of the Survey Respondents}

There was a total sample size of 261 responders with 117 completed surveys. The majority of responders were female (159 females (78\%) and 44 males (22\%)). All responders

provided care to individuals with PCOS as a physician $(n=138,66 \%)$, dietitian/nutritionist $(n=46$, $22 \%)$, or other specialty $(n=51,22 \%)$ (Table 1$)$. Sixty-six percent $(n=135)$ provided care to PCOS patients in a hospital or clinic setting, but $45 \%(n=92)$ practiced out of a private office, $(n=17) 8 \%$ in a research facility, and $(n=9) 4 \%$ in a different outlet. Input from urban setting providers $(n=98)$ made up $70 \%$ of the responses, followed by a suburban $(n=33,23 \%)$ and then rural locations $(n=9$, $6 \%)$. Survey responses came from across the world with $64 \%$ from the United States $(n=117)$ and $36 \%$ from outside the USA ( $n=67)$ based on self-reported locations. A summary of demographic information can be seen in Tables 3-5. View regional distribution of responders in Figures 2 and 3. 
Table 3: Demographic characteristics of provider specialty - multiple selections possible $(\mathrm{n}=210)$

\begin{tabular}{|c|c|c|}
\hline Specialty & \# Selected & \% Selected \\
\hline Physician & 138 & 66 \\
\hline Dietitian/Nutritionist & 46 & 5 \\
\hline Fertility Specialist & 11 & 4 \\
\hline Researcher & 8 & 3 \\
\hline Midlevel Providers (NP, PA) & 7 & 3 \\
\hline Educator/Counselor & 6 & 1 \\
\hline Lab Tech & 3 & 1 \\
\hline Psychologist & 2 & 1 \\
\hline Exercise Physiologist & 2 & 1 \\
\hline Physical Therapist & 2 & 0 \\
\hline Nurse & 1 & 4 \\
\hline Other & 9 & \\
\hline
\end{tabular}

Table 4: Breakdown of physician responders' area of expertise - multiple selections possible $(n=115)$

\begin{tabular}{|c|c|c|}
\hline Specialty & \# Selected & \% Selected \\
\hline General Pediatrics & 7 & 32 \\
\hline Adolescent Medicine & 37 & 35 \\
\hline Endocrinology & 40 & 30 \\
\hline Gynecology & 35 & 2 \\
\hline Integrative or Naturopathic Medicine & 2 & 1 \\
\hline Dermatology & 1 & \\
\hline
\end{tabular}


Table 5: Demographic characteristics of survey respondents

\begin{tabular}{|c|c|c|}
\hline \multicolumn{1}{|c|}{ Demographics } & \# Selected & \% Selected \\
\hline Sex & & 22 \\
\hline Female & 44 & 78 \\
\hline Setting for Care $\quad$ Hospital or Clinic & 159 & 66 \\
\hline Private Office & 135 & 45 \\
\hline Research Facility & 92 & 8 \\
\hline Other & 17 & 4 \\
\hline Population Setting & 9 & 70 \\
\hline Urban & 98 & 23 \\
\hline Suburban & 33 & 6 \\
\hline Rural & 9 & 1 \\
\hline Other & 1 & 36 \\
\hline Location & & \\
\hline United States & 117 & \\
\hline
\end{tabular}


Figure 2: USA regional breakdown of survey respondents $(\mathrm{N}=74)$

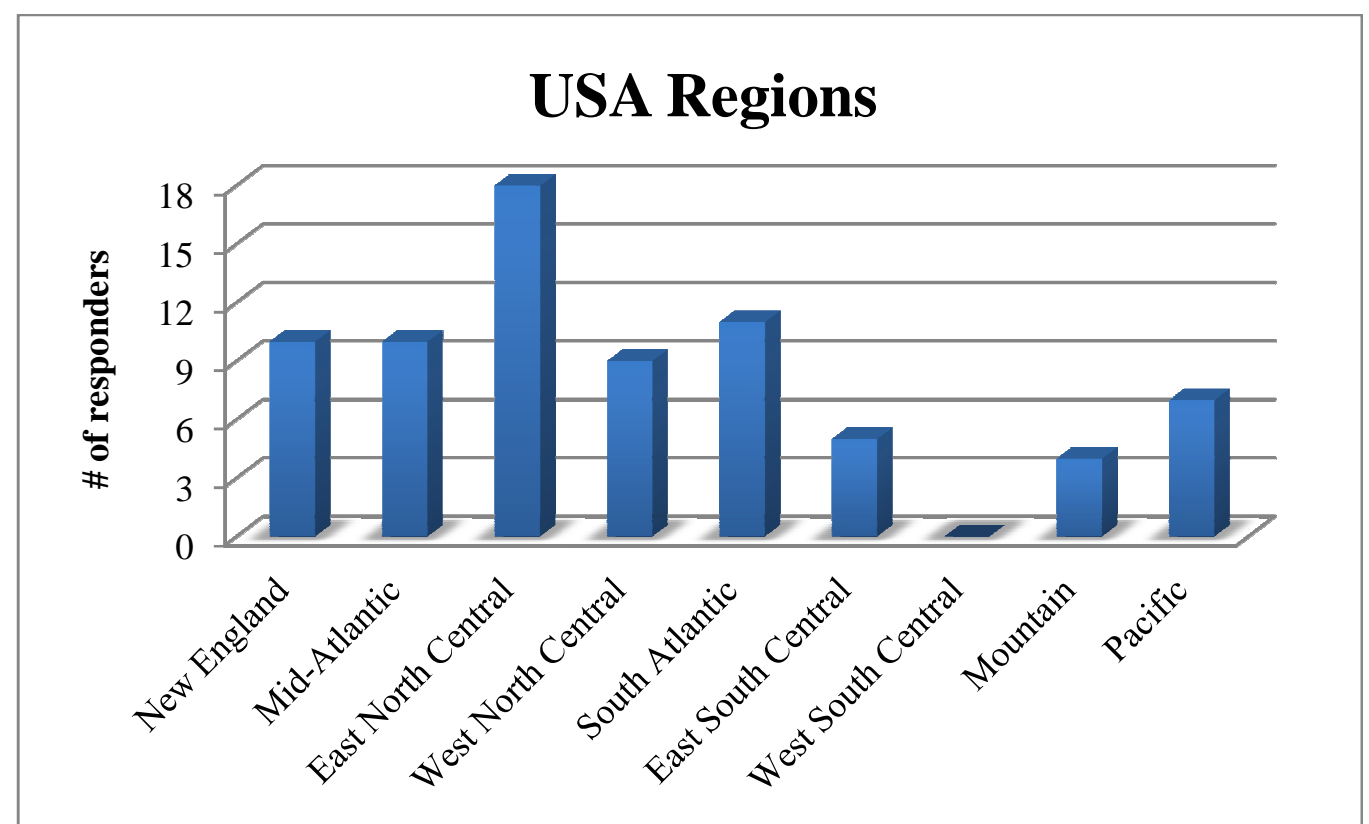

New England: MA, NH, VM,

MA, RI, CT

Mid-Atlantic: NY, PA, NJ

East North Central: WI, MI, IL, IN, OH

West North Central: MO, ND, SD, NE, KS, MN, IO
South Atlantic: DE, MD, DC, VA, WV, NC, SC, GA, FL East South Central: KY, TN, MS, AL

West South Central: OK, TX, AR, LA

Mountain: ID, MT, WY, NV, UT, CO, AZ, NM

Pacific: AK, WA, OR, CA, HI

Figure 3: World regional breakdown of survey respondents $(\mathrm{N}=41)$

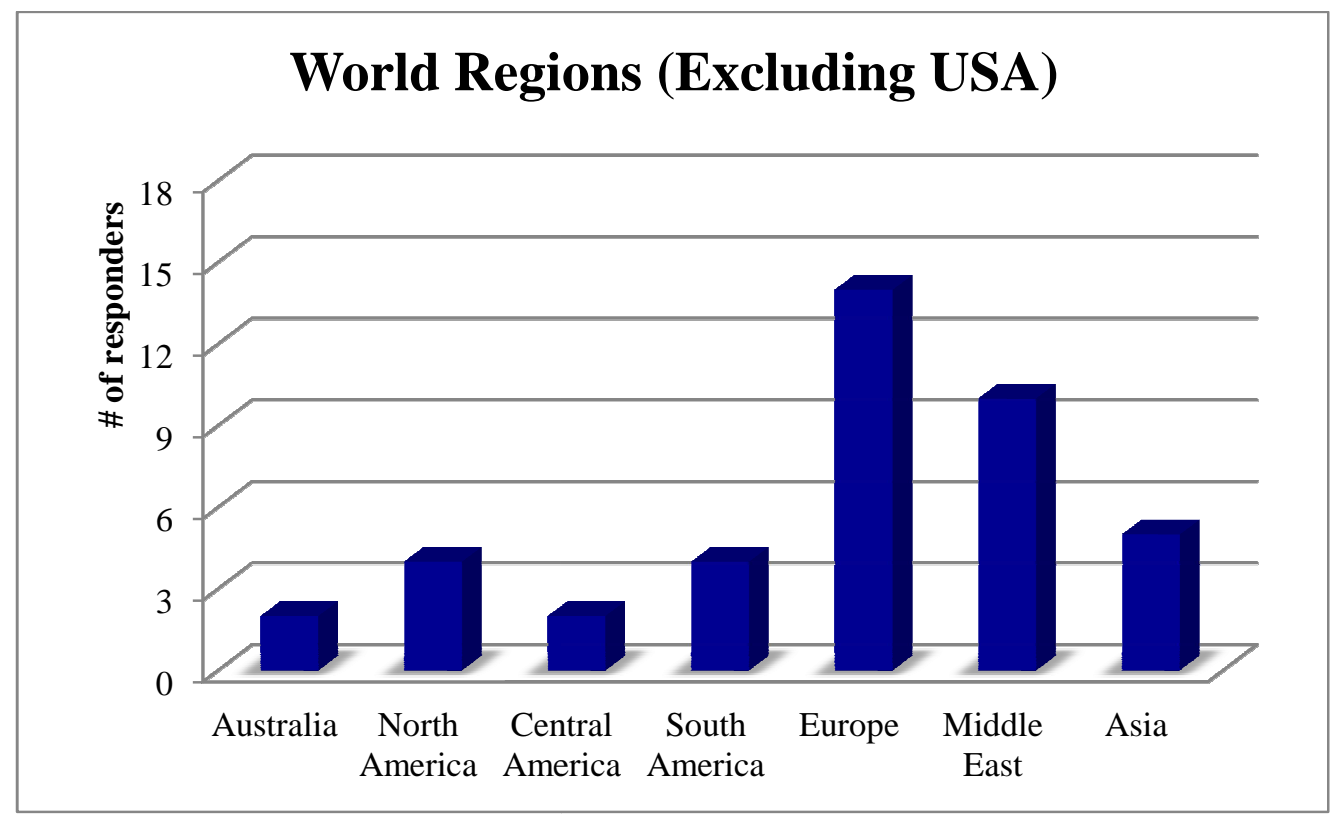




\section{Treatment Center Demographics}

The majority of clinics $(\mathrm{n}=79,56 \%)$ have been open for more than 10 years, with $19 \%$ $(\mathrm{n}=27)$ opening 5-10 years ago, $16 \%(\mathrm{n}=22)$ opening 2-5 years ago, and only 9\% $(\mathrm{n}=12)$ opening within the past 2 years. Fifty-nine percent $(n=79)$ of the responders treated PCOS in a multidisciplinary setting, defined as utilizing at least two health care providers from different specialties, whereas (n=56) $41 \%$ did not work in a multidisciplinary setting. Of the 140 responses, 79\% ( $\mathrm{n}=111$ ) stated their facility treated PCOS comprehensively as opposed to just one approach such as fertility. For the 29 clinics (21\%) that did not provide comprehensive care, 21 clinics listed their specialty and the most common focus was gynecology/fertility $(n=9,43 \%)$ followed by nutrition/weight loss $(\mathrm{n}=8,38 \%)$. Other responses included cardiology $(\mathrm{n}=1,5 \%)$, endocrinology $(\mathrm{n}=1,5 \%)$, and primary $(\mathrm{n}=2,10 \%)$. For those responders who were part of a multidisciplinary team the breakdown of specialties involved are listed below in Table 6 .

Table 6: Breakdown of specialty providers involved in multidisciplinary PCOS clinics ( $\mathrm{N}=132)$

\begin{tabular}{|c|c|c|}
\hline Specialty & \# Involved & \% Involved \\
\hline Dietitian/Nutritionist & 94 & 67 \\
\hline Physician & 89 & 48 \\
\hline Nurse & 63 & 35 \\
\hline Fertility Specialist & 46 & 28 \\
\hline Mid-Level Providers (NP, PA) & 37 & 28 \\
\hline Social Worker & 37 & 26 \\
\hline Psychologist & 34 & 23 \\
\hline Researcher & 30 & 15 \\
\hline Educator/Counselor & 20 & 11 \\
\hline Physical Therapist & 14 & 11 \\
\hline Other & 14 & 8 \\
\hline Exercise Physiologist & 11 & \\
\hline
\end{tabular}




\section{About the Patients}

The majority of clinics, $51 \%(\mathrm{n}=62)$, reported having less than 10 PCOS patients that are seen by any member in the facility each week; 30\% $(n=37)$ reported seeing 10-20 PCOS patients, $11 \%(\mathrm{n}=13)$ reported seeing $20-30$, and $8 \%(\mathrm{n}=10)$ reported seeing over 30 patients in an average week. When assessing the number average number of new patients that are seen for PCOS treatment on an annual basis in theses clinics, the majority of clinics $(n=121,58 \%)$ treated less than 50 each year; $24 \%(n=50)$ clinics saw 50-100 new patients, $10 \%(n=20)$ saw $100-150,2 \%(n=5)$ saw 150-200, and 5\% (n=11) saw over 200 new patients annually. The average youngest age of patients $(n=124)$ treated was reported to be 16.27 years old $($ median $=15$, mode $=12$, standard deviation $=5.526)$. The average oldest $(n=120)$ was 36.73 years old $($ median $=40$, mode $=40$, standard deviation $=11.593$ ). The greatest percentage of their patients were reported to be obese or overweight with a combined average of $69.78 \%$ of patients and the average reported breakdown is in Table 7.

Table 7: BMI category of patients treated for PCOS by survey responders $(\mathrm{N}=144)$

\begin{tabular}{|c|c|c|c|c|}
\hline BMI Category & Minimum \% & Maximum \% & Average \% & $\begin{array}{c}\text { Standard } \\
\text { Deviation }\end{array}$ \\
\hline Underweight: $<18.5$ & 0 & 15 & 2.17 & 3.78 \\
\hline Normal: $18.5-24.9$ & 0 & 50 & 13.13 & 12.21 \\
\hline Overweight: $25-29.9$ & 0 & 90 & 33.07 & 22.93 \\
\hline Obese: $>30$ & 0 & 100 & 36.71 & 27 \\
\hline
\end{tabular}

The diagnostic criteria used to diagnose PCOS by the survey responders was similar between teenagers and adults - see Figure 4. The most common diagnostic criteria used with teenage patients was the 2003 Rotterdam Criteria with 52\% (n=62), followed by the 2006 Androgen Excess Society (AES) criteria with 30\% (n=36), the 1990 National Institute of Health 
(NIH) criteria with $28 \%(n=33)$, and other with $16 \%(n=19)$. Responses for "other" used for teenagers included using a combination of NIH 1990, ROT 2003, and AES 2006 (n=4); ACOG $(n=1)$; or minor changes in the ultrasound method.

The most common diagnostic criteria used with adult patients was also the 2003 Rotterdam Criteria with $53 \%(n=55)$, followed by the 2006 AES criteria with $26 \%(n=27)$, the $1990 \mathrm{NIH}$ criteria with $19 \%(n=20)$, and other with $15 \%(n=15)$. Responses for "other" for adults using a combination of NIH 1990, ROT 2003, and AES $2006(n=1)$; ACOG $(n=1)$; or a consensus between ASRM and ESHRE.

Figure 4: Breakdown of PCOS diagnostic criteria used by the survey respondents

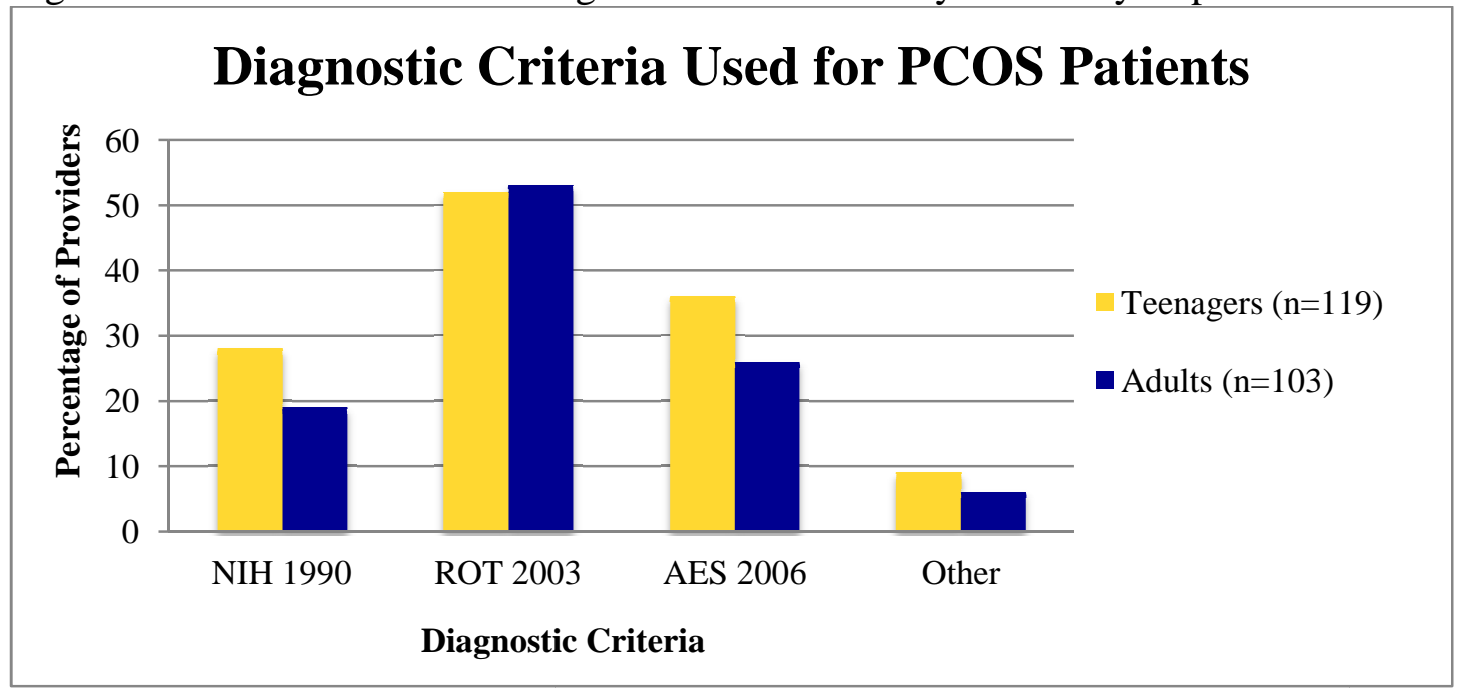

\section{Existing Clinic Outcomes}

Responders ( $n=88)$ were asked to list the top one or two items that their facility could improve upon. The most common theme identified in the responses, at $34 \%(n=30)$, was to incorporate more multidisciplinary involvement with more integration and/or communication. The second most popular theme, with $30 \%(\mathrm{n}=26)$ was to expand on nutrition and/or exercise programs to support weight loss. Improving or eliminating access barriers that prevent treatment of patients 
$(\mathrm{n}=9,10 \%)$ were also common themes. The three most common access barriers were identified to be patient wait-time $(n=5,56 \%)$, cost $(n=2,22 \%)$, and health insurance $(n=2,22 \%)$.

Responders ( $\mathrm{n}=87$ ) were also asked to list the top one or two items that their facility does well. The two most common responses, with $(n=18) 21 \%$ of responders each, were the treatment/management of symptoms and nutrition/lifestyle changes; $20 \%$ of responders $(n=17)$ stated patient education/counseling. With $17 \%$, multidisciplinary collaboration with other providers was the fourth most common theme identified $(n=15)$.

Responders rated their practice setting on the following criteria: retention rate, waiting time, prognosis outcomes, weight loss success, fertility outcomes, and patient satisfaction.

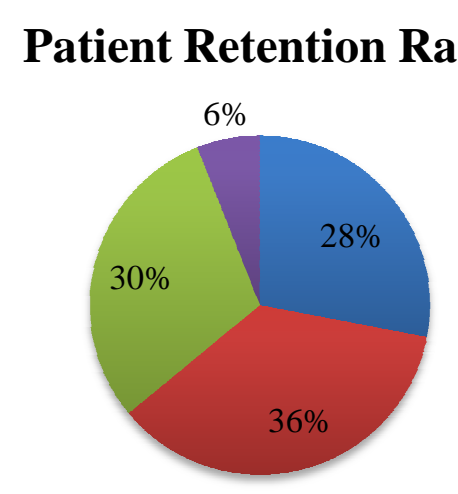

$\mathrm{N}=134$

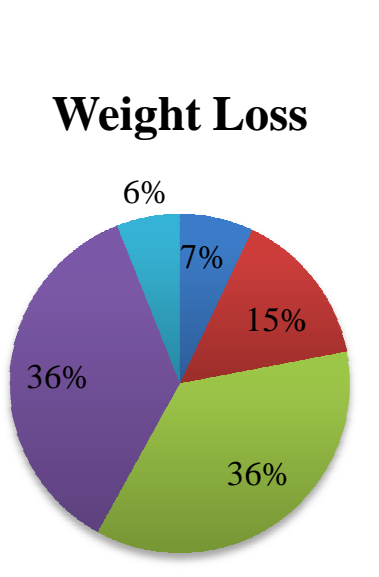

$\mathrm{N}=134$
Wait Time

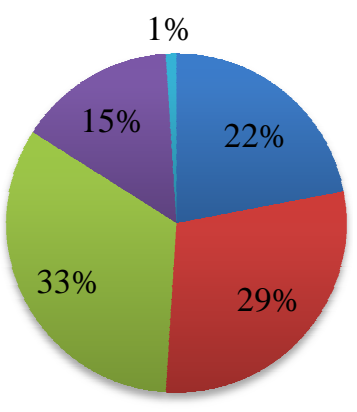

$\mathrm{N}=134$

\begin{tabular}{|l|}
\hline Excellent \\
Very Good \\
Good \\
Fair \\
Poor \\
\hline
\end{tabular}

\section{PCOS Prognosis}

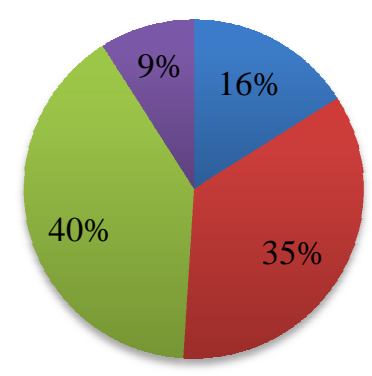

$\mathrm{N}=130$

\section{Fertility Outcomes}

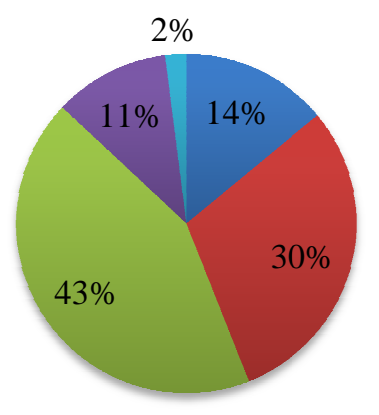

$\mathrm{N}=102$ 
The majority of respondents $(n=98,70 \%)$ reported that their PCOS treatment facility does not collect patient satisfaction data, where only $43(30 \%)$ do collect patient satisfaction data to any extent $(\mathrm{N}=141)$. For the clinics that reported collecting patient satisfaction data, the satisfaction level was generally positive.

Figure 6: Patient satisfaction data as self-reported from survey respondents $(\mathrm{N}=40)$

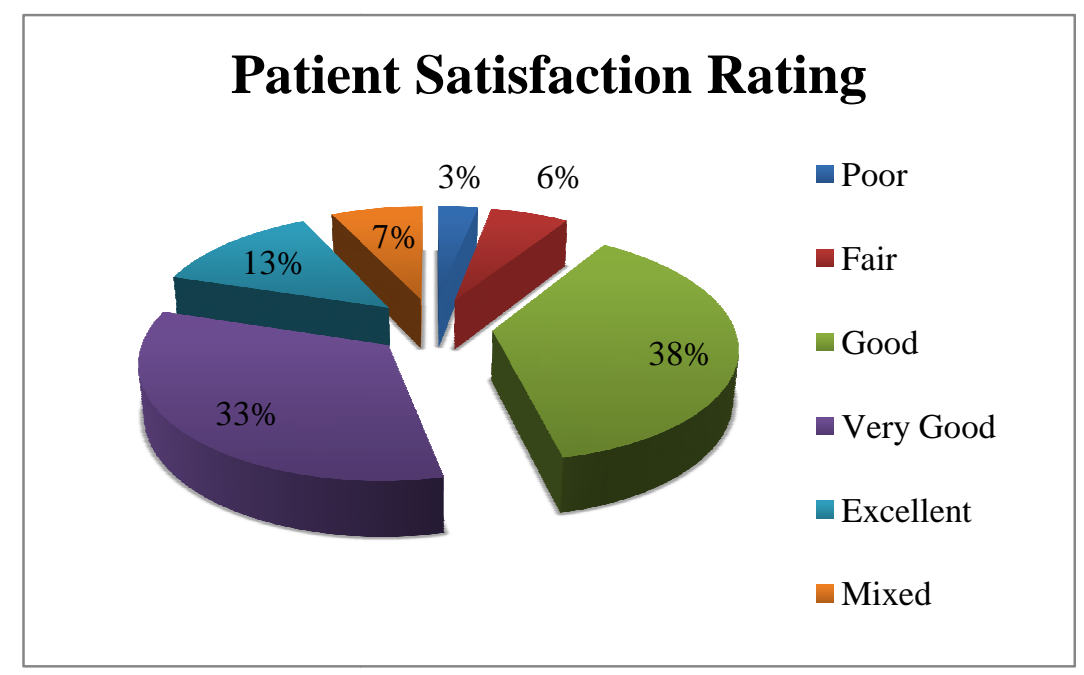

\section{Future Implications}

The most common potential barriers to future multidisciplinary clinics noted by responders $(n=76)$ were: money and resources $(n=23,30 \%)$, insurance/reimbursement $(n=20$, $26 \%)$, difference of opinions $(n=12,16 \%)$, and time $(n=9,12 \%)$. When asked to discuss the potential advantages of multidisciplinary PCOS clinics the most common responses $(n=82)$ were:

- Ability to provide more comprehensive and integrated care to address all aspects of PCOS $(\mathrm{n}=26,32 \%)$

- Better results/long-term care outcomes $(\mathrm{n}=12,18 \%)$

- All-in-one location $(\mathrm{n}=12,15 \%)$

- Greater convenience/efficiency $(\mathrm{n}=12,15 \%)$

- Allows for better coordination/communication/collaboration between providers $(\mathrm{n}=12$, $15 \%)$

- Increased access to more disciplines $(\mathrm{n}=8,10 \%)$ 
In order to determine the perceived benefits and importance of the involvement of specialties in future multidisciplinary clinics, responders were asked to rate the following providers.

Table 8: Ideal involvement of specialties in future multidisciplinary PCOS clinics $(\mathrm{N}=113)$

\begin{tabular}{|c|c|c|c|c|c|c|}
\hline Specialty & N & $\begin{array}{c}\text { Highly } \\
\text { Involved }\end{array}$ & Involved & Neutral & $\begin{array}{c}\text { Occasionally } \\
\text { Involved }\end{array}$ & $\begin{array}{c}\text { Never } \\
\text { Involved }\end{array}$ \\
\hline Endocrinologist & 109 & 48 & 36 & 6 & 7 & 3 \\
\hline Gynecologist & 110 & 45 & 43 & 5 & 5 & 2 \\
\hline Physician (Other) & 95 & 20 & 42 & 21 & 13 & 4 \\
\hline Dietitian/Nutritionist & 110 & 59 & 30 & 6 & 3 & 2 \\
\hline Psychologist & 105 & 11 & 45 & 21 & 15 & 8 \\
\hline $\begin{array}{c}\text { Mid-Level Providers } \\
\text { (NP, PA) }\end{array}$ & 90 & 17 & 31 & 29 & 11 & 11 \\
\hline Nurse & 96 & 19 & 32 & 30 & 15 & 4 \\
\hline Exercise Physiologist & 95 & 18 & 40 & 18 & 9 & 15 \\
\hline Fertility Specialist & 97 & 30 & 33 & 14 & 13 & 9 \\
\hline Social Worker & 93 & 10 & 25 & 31 & 22 & 13 \\
\hline Physical Therapist & 90 & 6 & 24 & 30 & 20 & 20 \\
\hline
\end{tabular}

\section{ii. Focus Group Results}

The purpose of conducting a series of focus groups was to further explore the topic of the utilization of registered dietitians for nutritional interventions for patients with PCOS and gain insight on the accessibility along with barriers and practicality.

\section{Participant Demographics}

The focus group participants included health care providers that fit in to one of the three following categories: registered dietitians, physicians, and other practitioners. The other practitioners consisted of one licensed nutritionist/certified nutrition specialist from functional medicine and a health and medical psychologist. All providers treated patients with PCOS on a 
regular basis and had between 7-25 years of experience. We conducted a series of focus groups via teleconferencing for a total of nine participants. The focus groups consisted of providers from similar backgrounds (i.e. physicians were only paired with physicians) to promote cohesiveness ${ }^{63,64}$ and compatibility ${ }^{65,66}$ as much as possible. Of the nine total participants, two were male and seven were females. We spoke with three physicians, which included two pediatric endocrinologist and one internal medicine/adolescent medicine physician, four registered dietitians, one health psychologist, and one licensed nutritionist/certified nutrition specialist. These providers primarily work in large metropolitan spread across the United States. The majority $(n=6)$ of participants worked in multidisciplinary facilities where they shared a location with other types of providers; where as the remaining three providers were solo providers who were in their own practice facility. Nearly all the providers in the focus group were very well-versed in PCOS and had been working directly with patients with PCOS for a range of 6-25 years.

\section{Describe Any Nutritional Interventions That You Provide To Your Patients.}

\section{$\underline{\text { Physicians }}$}

The majority of physicians that participated in this series of focus groups, reported collecting a basic diet history, determining food frequencies (of sugary beverages, dairy, etc.), and briefly touching on nutrition education in their initial visits with patients. These physicians also reported referring the majority of their patients to a dietitian for individualized counseling based on the interventions the patient is receiving. The majority of physicians reported that they are outliers in the fact that they focus heavily on nutrition in primary care and really value the importance where that is not typical amongst physicians. 


\section{$\underline{\text { Dietitians }}$}

All of the dietitians and other nutrition professionals that participated in the focus group series reported conducting intake assessments, providing the bulk of the nutrition education, and recommending a variety of individualized treatments depending on the patients' co-morbidities and goals. The dietitians are focusing on lifestyle interventions to manage their health, assessing food coping mechanism, and exploring emotional or disordered eating habits. The dietitian will also work with the patients to make sure that all patients are also seeing a physician who specializes in PCOS so that they will be medically managed correctly. One provider stated "Most of these women feel like they are not being listened to by the medical community specifically a lot their doctors just tell them to 'eat less, exercise more' but (are) not explaining to them...how their insulin levels are really effecting everything else that is going on with their bodies." This was a common theme that was addressed frequently that a large part of these nutritional interventions include helping the patient fully understand their condition and the interventions they are undergoing to make the patient feel like they are in control of their health, which makes them more likely to make changes.

When looking at the specific options the dietitians offer there were a variety of common approaches that were discussed. One clinic works to reach every PCOS patient with nutritional interventions by reducing the financial burden by offering group consultations and free monthly support groups to increase accountability in addition to the standard individual consultations. The frequency of visits with dietitians varied greatly depending on the patients and their condition, their insurance or financial situation. For example if a patient is dealing with emotional eating they will try to see that patient more often and one dietitian reported seeing the best results when she sees patients at least once or twice a month. In one clinic that is 
multidisciplinary and fully dedicated to PCOS, the patients always have the option to see a dietitian and if there was a dietary change that was recommended at the previous visit then the $\mathrm{RD}$ will be involved in the follow-up appointment.

As far as the specific interventions offered the most commonly discussed themes were centered around managing insulin resistance and the majority of these dietitian suggested low glycemic index diets, focusing on carbohydrate consistency, and reducing the size of carbohydrate portions. Another common theme was the focus on general healthy eating guidelines to reduce the risk of heart disease as well as improve their overall health. Another theme mentioned was the correction of any nutrient deficiencies that may be common with the medications typically prescribed for PCOS. Multiple dietitians mentioned the potential benefits of sensible dietary supplementation, which included things like vitamin D, myo-inositol, and others that have research supporting positive benefits with little or no side-effects. A few dietitians mentioned the importance for RDs of recognizing the value of dietary supplements and feel that physicians may value a provider that understands supplements and has that expertise. One nutrition profession stated "It can be very easy to overwhelm patients so it is beneficial to have a focused agenda and not try to cover everything in one visit." Dietitians also frequently mentioned the high need for individualized treatment because each patient will have different goals and concerns and nutrition professionals need to gear the focus to match theirs because even though providers can see the big connection and integration as a whole they might not see it that way. The role of the RD for patients with PCOS is really to educate the patients about small changes without overwhelming them and let the patients know that as a health care provider, you are not there to lecture them but to help them understand their condition and education them with compassion to better their health. 


\section{$\underline{\text { Health Psychologist }}$}

These providers reported often seeing patients who are very frustrated because they have read or heard so many different things about trying a variety of elimination diets and supplements that they end up frustrated or overwhelmed and that is really where health psychologist identified her role to be. The health psychologist reported that she emphasizes motivational interviewing, explores emotional or mindless eating habits, and really works with patient to facilitate that change according to the recommendations and education given by the dietitian. She always pairs the patients with a dietitian and helps them find what works for them so that they feel "like they are being proactive." Health coaches were reported being involved in one of the clinics and their role is to similar in the sense that they are there to assist patients with making the changes suggested by the nutrition professionals and to facilitate the behavior changes.

\section{How are Dietary Interventions and Patient Care Communicated Between Providers?/ What are the Potential Pros and Cons of Communication?}

When looking at the communication that occurred through the providers in the focus groups it did vary based on their practice setting. Overall, the providers who were located in multidisciplinary care centers, where the dietitian and physician were in the same facility, there was more verbal communication and integration of care. For some participants, being in the same location allowed the physician to speak with the dietitians before and after they saw a patient and meet in a shared conference space with all providers at the time of the patient visit. Other clinics that included a dietitian and physician had less communication because of the dynamic that the dietitian was brought in as an independent provider that solicited her services to reproductive endocrinologists who are very busy with the fertility aspect that there is no communication outside of the progress notes. There was very little formal case management 
meetings that was reported in the focus groups except for one clinic who held weekly case management meetings with all providers for their PCOS patients who also had eating disorders. Almost all of the participants stressed their desire for more communication between providers but it just does not work the way their clinic is set up now but they do feel it. One provider stated "In an ideal world, there would be PCOS treatment clinics all around the world and all the providers would have the opportunity to converse about each patient."

For those practitioners who are more solo providers there was no face to face communication and the only communication was via email and progress notes and they did face patient confidentiality barriers for discussing that patients care via email with other providers. For these providers where they are not all located in the same facility the treatment is "really piece milled out, which limits the opportunity for communication." One dietitian stated that once the doctors refer a patient to see her and she is actively treating that patient then the physician stops paying attention to diet because they know the dietitian is focusing on that but more integration and reinforcement can always help. The dietitians not located in multidisciplinary facilities stated that although it is not ideal, "the way we handle communication is still effective." "Communication is important in any case but I think it really helps support the patients so they know that we are all on the same page and the doctors can reinforce behaviors such as eliminating soda and checking in to see if the patient is compliant and to discuss how that it going and to provide extra support to the patient."

\section{When Do You Think Dietary Intervention is Warranted for a Patient With PCOS?/ How Does this Differ For Patients Depending on BMI Status?}

\section{When is Dietary Intervention Warranted?}

All providers involved in the focus group agreed and stated that it is always important to discuss nutrition and provide nutrition counseling and that they are equally important for all 
patients with PCOS regardless of weight or BMI status. The majority of providers felt that immediately upon on diagnosis of PCOS patients should meet with a dietitian for diet and lifestyle intervention because across the literature that is considered to be the first line treatment for PCOS. Providers stressed this importance and thought that diet needed to be addressed at every visit with every patient. One provider stated that PCOS should be treated with the same importance as any other chronic disease.

Why is Dietary Intervention Warranted?

As previously mentioned, diet and lifestyle intervention is considered the first line treatment for PCOS therefore it should be warranted for every PCOS patient. One of the most common themes addressed around the reasons PCOS patients need to see a dietitian was insulin resistance, which does occur frequently in lean women with PCOS as well. Women with PCOS who are overweight and obese are typically more symptomatic making them "more obvious referrals" but it important to remember that PCOS carries an increase risk for developing diabetes, heart disease, endometrial cancer even in lean PCOS. Another common theme across all providers was that lean PCOS patients could still be very malnourished and have a poor diet that will impact them later on in life.

What is Actually Happening?

Although all providers agreed that nutritional interventions are important for even women with PCOS, not all women with PCOS are getting lifestyle interventions. The physicians all agreed that not all patients with PCOS are typically seen by a dietitian and those who are lean are often overlooked and not given the same access to referrals. One solo provider stated that she rarely sees lean PCOS in her practice because they are just not referred there and the overweight and obese PCOS patients are the ones who spend the most time with the dietitians. One provider 
summed up this topic by saying "Women with lean PCOS are highly overlooked by the medical community because they don't think nutrition can help them because they are already thin even though a low glycemic index diet has been shown to increase ovulation and manage insulin abnormalities. I have seen plenty of lean PCOS women and dietary intervention absolutely helps!"

\section{How Accessible are Nutritional Interventions for the Majority of PCOS Patients?}

When the providers were asked how accessible they felt that nutritional interventions were for the majority of PCOS patients, providers unanimously agreed that overall, they are not very accessible. Factors that determine accessibility that were mentioned included their location, the willingness of physicians to refer out, and their insurance coverage or financial situation. Providers stressed that the biggest barriers are most likely insurance coverage and physicians that do not refer out because they do not see the benefits of nutritional interventions. One provider mentioned that without physician referrals, "It does not occur to most patients to seek out a dietitian to visit on their own." Of the participants in the focus group, one clinic only has access to a dietitian for initial consultations and then any follow-ups must be done at a different facility due to funding and as a result they see a lot of drop off for any follow-ups. Another facility stated the only way they are able to have a dietitian present in their clinic is because they also have a large diabetes program that covers the funding. It is important to address that these providers feel that dietitians in general are more accessible but there are just not a lot of dietitians who are well versed and experienced with PCOS. This is a very frustrating thing for providers and patients to be able to located providers that understand PCOS. 


\section{What are some of the Challenges for Getting Dietitians More Involved with the Treatment of PCOS?}

$\underline{\text { Insurance }}$

The most common theme heard throughout the series of focus groups across multiple questions was the insurance and reimbursement limitation for dietitians. Providers across the board stated that most HMOs and insurance companies will not cover nutritional counseling or will limit the number of visits to two-three per year, which does not allow adequate time to get the maximum benefit. The amount of nutritional counseling that is typically covered varies from state to state and does increase when PCOS patients also have conditions such as diabetes, which are covered more commonly. It was mentioned that insurance companies often times do not see a connection between nutrition and infertility. Unfortunately, when dietitians are not covered by insurance this becomes a huge cost barrier to patients and an issue for dietitians because they are not being reimbursed. One physician said "we are not able to effectively use dietitians because of the lack of insurance coverage."

\section{$\underline{\text { Lack of Education }}$}

Another common theme addressed primarily by nutrition professionals and health psychologist is that the majority of dietitians know very little about PCOS, let alone how to treat it. The dietitians stated that PCOS is not covered in their education and dietitians have often never heard of it. One provider said "There is no certification for PCOS so there is nothing across the board that provides a certain protocol for treatment. There are a few diagnostic tools but nobody has really agreed on which one to use." The limited training for RDs on PCOS is a huge problem and the profession needs to find a way to embrace this issue. It is very frustrating for patients when they go see a clinician that is not familiar with PCOS and they just get told the same information that does not help them. 


\section{Lack of Physician Referrals}

One of the most discussed themes was the lack of physician referrals for nutrition interventions with PCOS patients. One potential reason for physicians not referring as often as they should is the lack of insurance coverage. Most physicians stated that they feel more providers would be happy to refer patients if it was covered by insurance. They also stated physicians are likely not referring as often because of the limited access to dietitians who are experienced with PCOS. Physicians need to be made aware that there are specialists out there who can make a difference.

Another common theme across the series of focus groups was that physicians often times are quick to write patients off as uninterested or noncompliant with lifestyle interventions. One physician even stated that many physicians tend to not push lifestyle interventions because they do not have a lot of confidence that it will make a difference in the majority of patients expect the very highly motivated ones which he feels "are easy to pick out." This provider stated having similar thoughts and prior this focus group he has always viewed the dietitian as an additional service their clinic providers instead of an integral role in treatment. Unfortunately, the providers who have these beliefs are likely unaware of potential benefits when the nutritional interventions are done as an integral component of treatment with experienced providers. Another provider quoted "I just had a patient tell me that a doctor was just throwing people on medication (insulin sensitizers) instead of also referring to a dietitian because he believes they won't follow through, so he doesn't even try."

Another common theme that provides rational why physicians may not refer patients for nutritional counseling is that the physicians are so focused on intensely managing the medications and treatments that they feel they have treatment covered. Providers stated that this 
concept is commonly seen with reproductive endocrinologist who are treating patients who are often very eager to get pregnant that they are going to follow the more traditional pharmacology route that dedicate that time to lifestyle intervention. Overall, physicians are thought to value pharmacology over lifestyle interventions because of the higher compliancy rate which leads to doctors feeling like that they treatment covered without any lifestyle interventions. One of the physicians in the focus group stated that "some physicians just lack appreciation for what dietitians do."

The providers across these focus group feel like physicians have not been educated on the importance of nutritional interventions and do not always see the value of referring out. A dietitian summed up a major problem when she stated, "I think it is an education process that we need to continue to educate dietitians, as well and physicians, that we can provide value for PCOS treatment because physicians are the gatekeepers."

\section{$\underline{\text { Lack of Follow-Through from Patients }}$}

Another persistent theme throughout the series was that there is an additional set of barriers that exist to explain why women with PCOS may not follow through with a dietitian referral from a physician. The most common sub-theme was the lack of insurance coverage. Additional barriers included the patients who are just not ready to make any changes. Regardless of how much a patient may be in need nutritional intervention, if they are not ready to make a change they will not follow through. Patients are often very overwhelmed and overload how many providers they already need to see that they really do not want to take on another component. It was also noted that the practicality of coordinating and scheduling another visit that requires additional time off from school or work could deter patients from seeing a dietitian. Providers stated that there is additional drop-off in following through with referrals when it 
requires a separate visits and then even greater drop-off when the dietitian is not located in the same facility. Another common sub-theme was that many of these women are so damaged from being lectured by health care providers about their weight without getting any help or explanation of their condition. It was mentioned frequently that these patients are often not treated with a lot of sensitivity so they are hesitant to see another provider for free of another poor relationship. Participants mentioned that many people feel there is a certain stigma or it may be viewed as punishment to be referred to a dietitian. Providers have heard they patients say that they feel like they already know the dietitian is going to tell them and they already know they "should eat carrots instead of a snickers bar" so why bother going because they do not understand that it goes beyond that.

\section{$\underline{\text { Various Challenges and Opinions }}$}

The providers in these focus groups had a variety of additional challenges that exist for patients with PCOS and getting those patients the treatments they needed. Multiple providers stressed the need for dietitian support for PCOS and that "PCOS is really calling for registered dietitians and can potentially increase the need for what we do and really help a lot of people." Having a good dietitian who understands PCOS and how to effectively counsel patients with PCOS can really be the gateway into proper medical care of that patient by gaining that patients trust they will trust you when you advise them to see an additional provider. One provider mentioned that PCOS is a difficult condition for internal medicine doctors because it is primarily treated in the outpatient setting and the majority of programs that deal with internal medicine physicians deal with inpatients so that could be one reason it is overlooked. When looking at PCOS as a whole, another barrier that was mentioned was that "the diagnosis of PCOS in an adolescent is very controversial and there are several groups out there that are looking in to 
different ways to define it in adolescents with various phenotypes and we may end up seeing that these phenotypes require different dietary approaches."

\section{Importance of Involving Dietitians}

One common theme discussing the importance of involving dietitians in the treatment of PCOS patients was that it is the only way most patients will get adequate lifestyle interventions and help understand their condition. It was mentioned that physicians should not be responsible for the bulk of the nutrition interventions because they have little to no training in nutrition and "they can only the experts on so many things." These education sessions take time and it is so much "more than just handing the patient a 1,200 kcal diet plan and some physicians do not realize that." Patients benefit from nutritional counseling so much more than handing out a diet plan because there is a large psychological and emotional component related to food that much be addressed. One provider stated "The dietitian providers the nutritional information and the psychologist really gets the change."

\section{Do You Feel Like Providers Know and Understand the value of Nutritional Interventions for PCOS Patients?/ What are Some of the Differences Between Different Types of Providers?}

\section{$\underline{\text { Physicians }}$}

The major theme identified in the responses of the series of focus groups was that overall, health care providers do not understand the clinical complexities and the role of nutritional interventions in PCOS very well. When looking at physicians overall, the majority of participants felt that there were very few physicians that understand the value of nutritional interventions for PCOS but the vast majority did not. The most common theme in regards to physicians that was mentioned was that the majority of physicians do not understand the depth that is required in nutritional interventions. A quote by the psychologist stated, "It is so much more than handing them a diet plan and telling them to exercise and lose weight; It is about 
trying to un-root deeply seeded behaviors that are tied to emotions." Another common theme that was mentioned was that physicians often feel like they have the condition handled with medication and they tend to value pharmacotherapy higher than they do lifestyle interventions because of perceived greater success and compliance. This being noted, it is predicted that physicians do not see the value in referring out unless there is a major issue with diabetes, food allergies, or possible severe obesity. A comment by a dietitian stated "Physicians often treat PCOS with birth control pills and tell them to lose weight and exercise but do not do the followup monitoring or refer them to dietitians." It was mentioned that physicians often do not understand that the risk of developing long-term implications such as diabetes, heart disease, high blood pressure, and infertility increase when PCOS is not treated in it's entirety (by including the lifestyle intervention). It was mentioned that a physician's comfort level of treating PCOS will differ by the type of patients you see and the way you treat PCOS will differ across the specialties.

\section{$\underline{\text { Registered Dietitians/Nutrition Professionals }}$}

The most common theme addressed when looking at the understanding of PCOS across nutritional profession was that the vast majority of dietitians do not have a lot of training or knowledge in PCOS. One provider summed it up by saying, "Nutrition professionals need to be a lot better at what we do in terms of understanding PCOS and letting other providers know that we need to be involved." Two dietitians described personal experiences presenting information at state and national conferences and being shocked at how many dietitians have never even heard of PCOS when they can play such an integral role in the treatment of PCOS. One quote stated, "PCOS is the most common endocrine disorder among reproductive age women and dietitians don't even know what it is. That is a big issue!" 


\section{Implications and Suggestions for Change}

Across the focus groups the common theme was the lack of understanding and with that comes many implications. The most common implication was that the better the clinician understands PCOS, the better they are able to treat it and the more value they will see in the role of the dietitians and nutritional counseling. A major concern that was addressed was that when providers that do not understand PCOS are providing care for PCOS and not getting positive outcomes then it reflects poorly on the entire profession leading to poor relationships between health care providers and the PCOS patients. When looking at ways to change the lack of understand among providers it was mentioned that the first step is to just get the conversation started at the primary care level and to help show physicians the added value to the patients progress when dietitians and health psychologist are involved. One physician strongly believes and expressed that every physician needs to be referring every PCOS patient to a dietitian. A dietitian stated that she feels that "often times nutrition counseling is treated like dermatology and it needs to be treated more like psychology."

\section{In Your Career Working With PCOS, Have You Seen Any Shift In Awareness or Interest of PCOS?/If So, Please Describe Any Changes You Have Observed?}

The general consensus between providers across the focus groups was that they all felt there is much more awareness and recognition in the medical community. Physicians noted that they are speaking with their residents about it more often and the other providers mentioned that there are now people who claim to have a specialty in PCOS, which did not used to be the case. Another common theme identified was the they are noticing more information in the lay press so more patients are actually being the ones to ask their doctors to be tested for PCOS. In terms of the general public, these providers felt that there were more support groups and websites geared to help these women. While awareness for PCOS seems to be increasing, it was heavily 
emphasized that many providers still do not understand it and the following comment sums up the general consensus by saying "In general, women's health is not as much on the forefront and because PCOS affects only women it's on the list; It's starting to get mentioned but it doesn't get the attention it deserves." 


\section{Chapter V: Discussion}

The current formative study investigated the opinions of heath care providers who frequently treat PCOS on potential implications for the role of dietitians in the multidisciplinary treatment of PCOS. The hypothesis regarding the benefits of specialized individualized, and multidisciplinary care was demonstrated. There were a variety of challenges preventing dietitians from being involved to the fullest capacity with the treatment of PCOS.

Our survey found that $71 \%$ of individuals involved with a multidisciplinary clinic involved a dietitian, but a study on UK dietitians who treated PCOS found that only $36 \%$ worked jointly with other health professionals. ${ }^{56}$ Because our study advertised assessing multidisciplinary PCOS, it is likely that our sample attracted a higher percentage of multidisciplinary providers than is truly representative. Our results were lower than the results of a study assessing clinical variability in approaches to PCOS via a similar Internet survey to the NASPAG membership when they found that $90 \%$ of physicians recommended diet modification/exercise for a first-line treatment done by Bonny et al. ${ }^{42}$ While our study included NASPAG list serve we also included other outlets for recruitment which results in a different demographic between the two studies. Bonny et al. had a sample of $64 \%$ gynecologist, and 34\% adolescent medicine where our survey consisted primarily of close to one-third of gynecologist, adolescent medicine, and endocrinologist. While this exact question differed between surveys it could potentially show that members of the NASPAG who completed the survey by Bonney et al. are in fact recommending lifestyle modifications but not actually referring out to dietitians. Although this theory is just speculation, it is supported by the other studies who saw very minimal interactions with the dietitians. ${ }^{55,56}$ Jeanes et al. found that only $17 \%$ of lean PCOS and $25 \%$ of overweight women with PCOS received dietary advice from their physician. ${ }^{56}$ 
The limited accessibility to dietitians was addressed in our focus groups and it was mentioned that when dietitians are in a separate facility and require a separate visit less patients will actually see the dietitians. This study was a retrospective chart review in 2010 by Bekx et al. that demonstrated that $43 \%$ of all new referrals saw the health psychologist, $66 \%$ saw the nutritionist, $69 \%$ the gynecologist, and 100\% saw the endocrinologist. These numbers are greatly higher than the percentages of patients reported in other studies ${ }^{55,56}$ and reflected in our focus groups most likely because of the multidisciplinary clinic facility design. This same clinic was assessed in another study by Geier et al. that looked at patient with a diagnosis of PCOS under the Rotterdam criteria and they found that those numbers had increased to where $100 \%$ still saw the endocrinologist, but now $60.9 \%$ saw the health psychologist, $75.5 \%$ saw the dietitian, and $70.9 \%$ saw the gynecologist. ${ }^{7}$ Again, these findings are much higher than the percentage of patients seeing the dietitian that are not seen in these comprehensive multidisciplinary clinics and that were expressed in our focus groups.

When looking at the benefits of dietitians in the treatment of PCOS there has only been one study that assessed this so far. Geier et al. conducted a study at the American Family Children's Hospital in Madison, Wisconsin and assessed the factors contributing to the initial weight loss among adolescents with PCOS. Geier et al. found that $71 \%$ of 110 patients returned for a followup visit at the clinic and of those 57\% (45/78) succeeded at losing weight (mean loss of 3.5kg) and a total of $70 \%$ demonstrated weight loss or weight stabilization between the initial and the followup visit with an interval of 4.5 month (1.5-12 month range). ${ }^{7}$ When looking at the factors that may have attributed to the weight loss they saw that when comparing the providers seen with the relationship of weight loss, seeing a dietitian approached significance $(\mathrm{p}=0.06)$. It was not until they looked at patients who had seen both, the dietitian and the health psychologist, that they saw a 
significant difference. The patients who did not see either provider reported a mean weight gain of $2.0 \mathrm{~kg} \pm 2.8 \mathrm{~kg}$ and the patient who saw both provider reported a loss of $1.3 \mathrm{~kg} \pm 4.2 \mathrm{~kg}(\mathrm{p}=0.02)$. Seeing these providers had more of impact than being prescribed metformin, which had no independent effect. ${ }^{7}$

Results of our study supported the conclusions found by Bekx et al. that PCOS is a complex and heterogeneous disorder that requires multidisciplinary treatment to manage patients in the most effective way and the roles of each provider were similar to the themes of our focus group. Bekx et al. stated that the health psychologist and the nutritionist included motivational interviewing as a way to focus in on small lifestyle changes that were consistent and that were most likely to lead to the most success. ${ }^{39}$

The most frequent specific dietary intervention reported in our focus groups with the focus on insulin resistance through glycemic index and altering carbohydrate consistency and portion sizes. This was the same result found by Jeanes et al. in a survey focused on dietitians in the UK who treated PCOS, who found that $78 \%$ recommended caloric restriction, and $77 \%$ recommended choosing lower glycemic index foods, and often in combination. ${ }^{56}$ Jeanes et al. found that the dietary advice given to lean women with PCOS focused predominantly on reducing the glycemic index, general healthy eating, and increased physical activity, which was similar to the results of our focus group. Similar findings were reported in Bekx et al. that the nutritionist focuses on improving insulin resistance with the avoidance of prolonged fast and fiber-rich carbohydrates and protein. $^{39}$

The results of our focus groups signified that access to nutritional intervention counseling is very limited for the majority of PCOS patients. Jeanes et al., who used a patient questionnaire to survey women in the UK with PCOS to determine where they receive their nutritional information, 
supported this finding. This study found that only $15 \%$ of patients with PCOS had ever seen a dietitian; that number was further reduced to just $3 \%$ for patients who had more than two appointments with a dietitian. This study did not report how many of these patients had ever seen a referral. Humphreys et al. ${ }^{55}$ conducted in-depth interviews with PCOS patients in the UK and found the only $26 \%$ of overweight or obese women with PCOS had ever received a referral to see a dietitian, which also supported our findings of the limited access and the limited number of physician referrals.

When assessing the differences in accessibility to dietitians and nutritional interventions in overweight and obese verse women with lean PCOS the focus group results found that lean PCOS is often overlooked and the obese PCOS cases are typically more symptomatic, making them more obvious referrals despite the perception of it being of equal importance. This findings are supported by Jeanes et al. who found that overweight women were more likely to receive dietary advice from a dietitian (21\%) than lean with 10\% and from the physicians, with $25 \%$ and $17 \%$ respectively. Humphreys et al. found that only the overweight women had been given dietary advice from their consultant endocrinologist; the patients rated this information as useful but very general and inadequate. ${ }^{55}$

Geier et al. looked at barriers that existed to prevented all of the patients in a multidisciplinary clinic setting to seeing the dietitian and health psychologist and found similar results to the barriers identified in the both phases of the current study. The number one barrier was the denial of access by referring HMOs or insurance providers followed by the patient refusing the visit due to the perceived stigma or they did not want to consider dietary interventions. Another barrier that was discovered by Geier that was not mentioned in our study was that some patients had a prior therapeutic relationship was a psychologist or psychiatrist that was not 
affiliated with this multidisciplinary clinic. It was noted in this study that there was a lack of perceived benefit from patients with PCOS that had a normal BMI, even though a few still had insulin resistance. ${ }^{7}$ This was similar to the concept addressed in the focus group that they already know what the dietitian is going to tell them or that they think because they are already lean diet changes won't help them. The study by Geier et al. was a retrospective study that had no consistent documentation for refusal reasons and it should be evaluated in a prospective study.

Our study noted that a major challenge to dietitians in the US in regards to treating PCOS, was the lack of focused PCOS education for dietitians. Jeanes et al., who found that amongst UK dietitians who treated PCOS only $34 \%$ reported feeling well informed of the PCOS literature, and $64 \%$ believed that there was insufficient evidence regarding the dietary management of PCOS in 2009, support this finding. ${ }^{56}$ The fact that only one-third of dietitians who see women with PCOS feel confident in their abilities to treat PCOS, supports the common focus group theme that there are very few dietitians across the board that understand PCOS.

One major barrier for dietitians that our study found was the lack in physician referrals. Potential reasons for the lack in referrals were noted to include the lack of confidence that physicians have that lifestyle intervention is beneficial. These findings were supported by a study by Foster et al. ${ }^{70}$ that was a random survey of physicians that found that $43 \%$ of the physicians surveyed think that most obese patients will not lose a significant amount of weight. The same survey also demonstrated that only $14 \%$ of those physicians believe that they are usually successful in assisting obese patients lose weight. Another study by Baillargeon et al. ${ }^{71}$ demonstrated that physicians are not comfortable discussing obesity and they believe it is not worth the time it takes. A study by Pelletier et al. looked to determine the proportion of obese women with PCOS who achieved clinically significant weight loss, defined as at least 5-10\% of their initial weight when 
provided with only regular follow-up by an endocrinologist, without an integrated multidisciplinary. This retrospective chart study did encourage participants to visit a dietitian but this was not recorded and the dietitian located in the clinic was only accessible to the women who had diabetes. The endocrinologist had 45-50 minute initial consultation and then follow-ups that lasted about 25 minutes. During these visits the physicians explained PCOS, nutrition education regarding basic healthy items, and emphasized physical activity, which were all reinforced during follow-up visits. This study found that over $40 \%$ of obese PCOS women lost $\geq 5 \%$ of their initial weight after at least six months of follow-up with an endocrinologist and $20 \%$ lost $\geq 10 \%$ after one year of follow-up. ${ }^{72}$ This should provide physicians with more confidence that lifestyle intervention is effective.

When assessing the importance of dietitians our focus groups conveyed that physicians should not be the ones fully responsible for dietary interventions because they lack the training and the time it takes to facilitate the change. Humphreys et al. noted that $80 \%$ of overweight PCOS patients found weight loss difficult and only $23 \%$ have had success on their current weight reduction diet. ${ }^{55}$ These patients received their information from the Internet or their endocrinologist whom they only saw twice a year, and remember that only $26 \%$ had ever been referred to a dietitian. ${ }^{55}$ A random survey of the American Medical Association in 2003 that was conducted by Pelletier et al. determined that only $49 \%$ of physicians felt competent in prescribing weight loss programs, which supports the theme identified in our focus groups that physicians do not have the proper education for nutritional interventions. ${ }^{72}$

Lifestyle intervention counseling is overlooked heavily in the treatment of PCOS and there are many challenges to incorporating dietitians but with improvements in education and insurance they can play an integral role in PCOS. Our study and the findings of others found that despite the 
fact that weight loss and weight maintenance are vital in the reducing of symptoms and long-term risk for PCOS, the general consensus is that the education and support given to these patients is inadequate. 


\section{Chapter VI: Summary and Conclusions}

PCOS is a complex condition that requires the expertise of multiple provider types to treat the syndrome in its entirety. Most providers agree that a multidisciplinary clinic would provide greater convenience, access to care, and ultimately lead to a better prognosis for patients with

PCOS. The perceived barriers that prevent clinics from becoming multidisciplinary would need to be well defined, but providers indicate enthusiasm for the opportunity to implement a multidisciplinary approach. 


\section{References}

1. Okoroh EM, Hooper WC, Atrash HK, Yusuf HR, Boulet SL. Prevalence of polycystic ovary syndrome among the privately insured, United States, 2003-2008. Obstet Gynecol.

2012;207(4):299.e1-299.e7. doi: 10.1016/j.ajog.2012.07.023.

2. March WA, Moore VM, Willson KJ, Phillips DI, Norman RJ, Davies MJ. The prevalence of polycystic ovary syndrome in a community sample assessed under contrasting diagnostic criteria. Hum Reprod. 2010;25(2):544-551. doi: 10.1093/humrep/dep399.

3. Knochenhauer ES, Key TJ, Kahsar-Miller M, Waggoner W, Boots LR, Azziz R. Prevalence of the polycystic ovary syndrome in unselected Black and White women of the Southeastern United States: A prospective study. J Clin Endocrinol Metab. 1998;83(9):3078-3082.

4. Kauffman RP, Baker VM, Dimarino P, Gimpel T, Castracane VD. Polycystic ovarian syndrome and insulin resistance in White and Mexican American women: A comparison of two distinct populations. Am J Obstet Gynecol. 2002;187(5):1362-1369.

5. Azziz R, Carmina E, Dewailly D, et al. The Androgen Excess and PCOS Society criteria for the polycystic ovary syndrome: The complete task force report. Fertil Steril. 2009;91(2):456-488. doi: 10.1016/j.fertnstert.2008.06.035.

6. Broekmans FJ, Knauff EA, Valkenburg O, Laven JS, Eijkemans MJ, Fauser BC. PCOS according to the Rotterdam consensus criteria: Change in prevalence among WHO-II anovulation and association with metabolic factors. BJOG. 2006;113(10):1210-1217. doi: 10.1111/j.14710528.2006.01008.x. 
7. Geier LM, Bekx MT, Connor EL. Factors contributing to initial weight loss among adolescents with polycystic ovary syndrome. J Pediatr Adolesc Gynecol. 2012. doi:

10.1016/j.jpag.2012.06.008; 10.1016/j.jpag.2012.06.008.

8. Lamb J, Closshey W, Huddleston H, Davis G, Zane L, Cedars M. A multidisciplinary polycystic ovarian syndrome (PCOS) clinic: A new model for care and research. Fertil Steril. 2007;88:S186S186.

9. Barber TM, McCarthy MI, Wass JAH, Franks S. Obesity and polycystic ovary syndrome. Clin Endocrinol (Oxf). 2006;65(2):137-145. doi: 10.1111/j.1365-2265.2006.02587.x.

10. Azziz R, Sanchez LA, Knochenhauer ES, et al. Androgen excess in women: Experience with over 1000 consecutive patients. The Journal of Clinical Endocrinology \& Metabolism. 2004;89(2):453-462. doi: 10.1210/jc.2003-031122.

11. Sirmans SM, Pate KA. Epidemiology, diagnosis, and management of polycystic ovary syndrome. Clin Epidemiol. 2013;6:1-13. doi: 10.2147/CLEP.S37559.

12. Shakerardekani Z, Nasehi AA, Eftekhar T, Ghaseminezhad A, Shaker Ardekani MA, Raisi F. Evaluation of depression and mental health status in women with polycystic ovary syndrome. Journal of Family \& Reproductive Health. 2011;5(3):67-71.

http://search.ebscohost.com/login.aspx?direct=true \&db=a9h\&AN=79449572\&site=ehost-live.

13. Coffey S, Bano G, Mason H. Health-related quality of life in women with polycystic ovary syndrome: A comparison with the general population using the polycystic ovary syndrome questionnaire (PCOSQ) and the short form-36 (SF-36). Gynecological Endocrinology. 2006;22(2):80-86. 
http://search.ebscohost.com/login.aspx?direct=true $\& d b=a 9 h \& A N=20426908 \&$ site=ehost-live. doi: $10.1080 / 09513590600604541$.

14. Mandrelle K, Kamath MS, Bondu DJ, Chandy A, TK A, George K. Prevalence of metabolic syndrome in women with polycystic ovary syndrome attending an infertility clinic in a tertiary care hospital in South India. Journal of Human Reproductive Sciences. 2012;5(1):26-31.

http://search.ebscohost.com/login.aspx?direct=true $\& \mathrm{db}=\mathrm{a} 9 \mathrm{~h} \& \mathrm{AN}=77707220 \&$ site=ehost-live. doi: 10.4103/0974-1208.97791.

15. Legro RS, Kunselman AR, Dodson WC, Dunaif A. Prevalence and predictors of risk for type 2 diabetes mellitus and impaired glucose tolerance in polycystic ovary syndrome: A prospective, controlled study in 254 affected women. J Clin Endocrinol Metab. 1999;84(1):165-169.

16. Amato MC, Galluzzo A, Finocchiaro S, Criscimanna A, Giordano C. The evaluation of metabolic parameters and insulin sensitivity for a more robust diagnosis of the polycystic ovary syndrome. Clin Endocrinol (Oxf). 2008;69(1):52-60.

http://search.ebscohost.com/login.aspx?direct=true $\& d b=a 9 h \& A N=32642418 \&$ site=ehost-live. doi: 10.1111/j.1365-2265.2007.03145.x.

17. Carmina E. Diagnosis of polycystic ovary syndrome: From NIH criteria to ESHRE-ASRM guidelines. Minerva Ginecol. 2004;56(1):1-6.

18. Garad R, Teede H, Moran L. An evidence-based guideline for polycystic ovary syndrome. Aust Nurs J. 2011;19(4):30-33.

http://search.ebscohost.com/login. aspx ?direct=true $\& d b=c 8 h \& A N=2011305148 \&$ site=ehost-live. 
19. Meurer LN, Kroll AP, Jamieson B, Yousefi P. Clinical inquiries. What is the best way to diagnose polycystic ovarian syndrome? J Fam Pract. 2006;55(4):351-2, 354.

20. Azziz R, Woods KS, Reyna R, Key TJ, Knochenhauer ES, Yildiz BO. The prevalence and features of the polycystic ovary syndrome in an unselected population. $J$ Clin Endocrinol Metab. 2004;89(6):2745-2749. doi: 10.1210/jc.2003-032046.

21. Lim SS, Norman RJ, Davies MJ, Moran LJ. The effect of obesity on polycystic ovary syndrome: A systematic review and meta-analysis. Obes Rev. 2012. doi: 10.1111/j.1467789X.2012.01053.x; 10.1111/j.1467-789X.2012.01053.x.

22. Futterweit W. Polycystic ovary syndrome: Clinical perspectives and management. Obstet Gynecol Surv. 1999;54(6):403-413.

23. Carmina E, Koyama T, Chang L, Stanczyk FZ, Lobo RA. Does ethnicity influence the prevalence of adrenal hyperandrogenism and insulin resistance in polycystic ovary syndrome? Am J Obstet Gynecol. 1992;167(6):1807-1812.

24. Asuncion M, Calvo RM, San Millan JL, Sancho J, Avila S, Escobar-Morreale HF. A prospective study of the prevalence of the polycystic ovary syndrome in unselected Caucasian women from Spain. J Clin Endocrinol Metab. 2000;85(7):2434-2438.

25. Razak F, Anand S, Vuksan V, et al. Ethnic differences in the relationships between obesity and glucose-metabolic abnormalities: A cross-sectional population-based study. Int J Obes (Lond). 2005;29(6):656-667. doi: 10.1038/sj.ijo.0802937. 
26. Dunstan DW, Zimmet PZ, Welborn TA, et al. The rising prevalence of diabetes and impaired glucose tolerance: The Australian diabetes, obesity and lifestyle study. Diabetes Care. 2002;25(5):829-834.

27. Zhu S, Wang Z, Heshka S, Heo M, Faith MS, Heymsfield SB. Waist circumference and obesity-associated risk factors among whites in the third national health and nutrition examination survey: Clinical action thresholds. Am J Clin Nutr. 2002;76(4):743-749.

28. Ogden CL, Carroll MD, Kit BK, Flegal KM. Prevalence of obesity in the United States, 20092010. NCHS Data Brief. 2012;(82)(82):1-8.

29. Davis SR, Knight S, White V, Claridge C, Davis BJ, Bell R. Preliminary indication of a high prevalence of polycystic ovary syndrome in indigenous Australian women. Gynecol Endocrinol. 2002;16(6):443-446.

30. Kumarapeli V, Seneviratne Rde A, Wijeyaratne CN, Yapa RM, Dodampahala SH. A simple screening approach for assessing community prevalence and phenotype of polycystic ovary syndrome in a semi-urban population in Sri Lanka. Am J Epidemiol. 2008;168(3):321-328. doi: 10.1093/aje/kwn137.

31. Azziz R, Marin C, Hoq L, Badamgarav E, Song P. Health care-related economic burden of the polycystic ovary syndrome during the reproductive life span. J Clin Endocrinol Metab. 2005;90(8):4650-4658. doi: 10.1210/jc.2005-0628.

32. Wijeyaratne CN, Balen AH, Barth JH, Belchetz PE. Clinical manifestations and insulin resistance (IR) in polycystic ovary syndrome (PCOS) among South Asians and Caucasians: Is there a difference? Clin Endocrinol (Oxf). 2002;57(3):343-350. 
33. Rodin DA, Bano G, Bland JM, Taylor K, Nussey SS. Polycystic ovaries and associated metabolic abnormalities in Indian subcontinent Asian women. Clin Endocrinol (Oxf). 1998;49(1):91-99.

34. Shannon M, Wang Y. Polycystic ovary syndrome: A common but often unrecognized condition. J Midwifery Womens Health. 2012;57(3):221-230. doi: 10.1111/j.15422011.2012.00161.x; 10.1111/j.1542-2011.2012.00161.x.

35. Moran C, Tena G, Moran S, Ruiz P, Reyna R, Duque X. Prevalence of polycystic ovary syndrome and related disorders in Mexican women. Gynecol Obstet Invest. 2010;69(4):274-280. doi: 10.1159/000277640.

36. Goodarzi MO, Quinones MJ, Azziz R, Rotter JI, Hsueh WA, Yang H. Polycystic ovary syndrome in Mexican-Americans: Prevalence and association with the severity of insulin resistance. Fertil Steril. 2005;84(3):766-769. doi: 10.1016/j.fertnstert.2005.03.051.

37. Diamanti-Kandarakis E, Kouli CR, Bergiele AT, et al. A survey of the polycystic ovary syndrome in the Greek Island of Lesbos: Hormonal and metabolic profile. J Clin Endocrinol Metab. 1999;84(11):4006-4011.

38. Azziz R, Dumesic DA, Goodarzi MO. Polycystic ovary syndrome: An ancient disorder? Fertil Steril. 2011;95(5):1544-1548. doi: 10.1016/j.fertnstert.2010.09.032.

39. Bekx MT, Connor EC, Allen DB. Characteristics of adolescents presenting to a multidisciplinary clinic for polycystic ovarian syndrome. J Pediatr Adolesc Gynecol. 2010;23(1):710. doi: 10.1016/j.jpag.2009.04.004. 
40. Broder-Fingert S, Shah B, Kessler M, Pawelczak M, David R. Evaluation of adolescents for polycystic ovary syndrome in an urban population. Journal of Clinical Research in Pediatric Endocrinology. 2009;1(4):188-193.

http://search.ebscohost.com/login.aspx?direct=true \&db=a9h\&AN=44451190\&site=ehost-live. doi: 10.4008/jcrpe.v1i4.50.

41. Sivayoganathan D, Maruthini D, Glanville J, M., Balen A, H. Full investigation of patients with polycystic ovary syndrome (PCOS) presenting to four different clinical specialties reveals significant differences and undiagnosed morbidity. HUM FERTIL (CAMB). 2011;14(4):261-265. http://search.ebscohost.com/login.aspx?direct=true \&db=c8h\&AN=2011365262\&site=ehost-live. doi: 10.3109/14647273.2011.632058.

42. Bonny AE, Appelbaum H, Connor EL, et al. Clinical variability in approaches to polycystic ovary syndrome. J Pediatr Adolesc Gynecol. 2012;25(4):259-261. doi: 10.1016/j.jpag.2012.03.004.

43. Ghosh D, Murphy C, Elsheikh M. A 2 year audit of the polycystic ovary syndrome (PCOS) clinic at the Royal Berkshire Hospital. Endocrine Abstracts. 2005;9:79.

44. Eldridge S, Murphy C, Elsheikh M. Audit of the polycystic ovary syndrome (PCOS) nurse led weight management clinic. 2007;13:P255.

45. Moran LJ, Pasquali R, Teede HJ, Hoeger KM, Norman RJ. Treatment of obesity in polycystic ovary syndrome: A position statement of the Androgen Excess and Polycystic Ovary Syndrome Society. Fertil Steril. 2009;92(6):1966-1982. doi: 10.1016/j.fertnstert.2008.09.018; 10.1016/j.fertnstert.2008.09.018. 
46. Moran LJ, Lombard CB, Lim S, Noakes M, Teede HJ. Polycystic ovary syndrome and weight management. Womens Health. 2010;6(2):271-83.

http://search.proquest.com.www.libproxy.wvu.edu/docview/229601598?accountid=2837. doi: http://dx.doi.org.www.libproxy.wvu.edu/10.2217/whe.09.89.

47. Moran LJ, Ko H, Misso M, et al. Dietary composition in the treatment of polycystic ovary syndrome: A systematic review to inform evidence-based guidelines. J Acad Nutr Diet. 2013;113(4):520-545. doi: 10.1016/j.jand.2012.11.018; 10.1016/j.jand.2012.11.018.

48. Teede HJ, Misso ML, Deeks AA, et al. Assessment and management of polycystic ovary syndrome: Summary of an evidence-based guideline. Med J Aust. 2011;195(6):S65-112.

49. Norman RJ, Davies MJ, Lord J, Moran LJ. The role of lifestyle modification in polycystic ovary syndrome. Trends in endocrinology and metabolism: TEM JID - 9001516. 2010.

50. Norman RJ, Homan G, Moran L, Noakes M. Lifestyle choices, diet, and insulin sensitizers in polycystic ovary syndrome. Endocrine. 2006;30(1):35-43. doi: 10.1385/ENDO:30:1:35.

51. Hutchison SK, Stepto NK, Harrison CL, Moran LJ, Strauss BJ, Teede HJ. Effects of exercise on insulin resistance and body composition in overweight and obese women with and without polycystic ovary syndrome. J Clin Endocrinol Metab. 2011;96(1):E48-56. doi: 10.1210/jc.20100828; 10.1210/jc.2010-0828.

52. Poehlman ET, Dvorak RV, DeNino WF, Brochu M, Ades PA. Effects of resistance training and endurance training on insulin sensitivity in nonobese, young women: A controlled randomized trial. J Clin Endocrinol Metab. 2000;85(7):2463-2468. doi: 10.1210/jcem.85.7.6692. 
53. Ross R, Dagnone D, Jones PJ, et al. Reduction in obesity and related comorbid conditions after diet-induced weight loss or exercise-induced weight loss in men. A randomized, controlled trial. Ann Intern Med. 2000;133(2):92-103.

54. Moran LJ, Ko HF, Misso MF, et al. Dietary composition in the treatment of polycystic ovary syndrome: A systematic review to inform evidence-based guidelines. Journal of the Academy of Nutrition and Dietetics JID - 101573920. 2013.

55. Humphreys L, Costarelli V. Implementation of dietary and general lifestyle advice among women with polycystic ovarian syndrome. The journal of the Royal Society for the Promotion of Health JID - 101499616. 2008.

56. Jeanes YM, Barr SF, Smith K FAU, Hart KH. Dietary management of women with polycystic ovary syndrome in the United Kingdom: The role of dietitians. Journal of human nutrition and dietetics: the official journal of the British Dietetic Association JID - 8904840. 2009(1365-277).

57. Stein K. Polycystic ovarian syndrome: What it is and why registered dietitians need to know. Journal of the American Dietetic Association JID - 7503061. 2006.

58. Seagle HM, Strain GW, Makris A, Reeves RS, American Dietetic Association. Position of the American Dietetic Association: Weight management. J Am Diet Assoc. 2009;109(2):330-346.

59. Morse J, Field P. Nursing research: The application of qualitative approaches. 2 nd ed. London: Chapman and Hall; 1996. 
60. Battaglia MP. Nonprobability sampling. In: Lavrakas PJ, ed. Encyclopedia of survey research methods. Thousand Oaks, Calif.: SAGE Publications; 2008:523-523-526.

http://dx.doi.org/10.4135/9781412963947.

61. Glaser B. Theoretical sensitivity: Advances in the methodology of grounded theory. Sociology Press: Mill Valley, CA; 1978.

62. Glaser B, Strauss A. The discovery of grounded theory: Strategies for qualitative research. New York: Aldine; 1967.

63. Cartwright D, Zander A. The nature of group cohesiveness. In: Cartwright D, Zander A, eds. Group dynamics: Research and theory. 3rd ed. New York: Harper and Row; 1968.

64. Terborg J, Castore C, DeNinno J. A longitudinal field investigation of the impact of group composition on group performance and cohesion. Journal of Personality and Social Psychology. 1976;34:782-790.

65. Haythorn W, Couch A, Haefner D, Langham P, Carter L. The behavior of authoritarian and equalitarian personalities in groups. Human Relations. 1956;9:57-74.

66. Sapolsky A. Effect of interpersonal relationships upon verbal conditioning. Journal of Abnormal and Social Psychology. 1960;60:241-246.

67. Holloway I, Wheeler S. Qualitative research for nurses. London: Blackwell; 1996.

68. Birks M, Mills J. Grounded theory: A practical guide. Thousand Oaks, CA: Sage; 2011:224. 
69. Braun V, Clarke V. Using thematic analysis in psychology. Qualitative Research in Psychology. 2006;3(2):77-101. http://www.tandfonline.com/doi/abs/10.1191/1478088706qp063oa. doi: 10.1191/1478088706qp063oa.

70. Foster GD, Wadden TA, Makris AP, et al. Primary care physicians' attitudes about obesity and its treatment. Obes Res. 2003;11(10):1168-1177. doi: 10.1038/oby.2003.161.

71. Baillargeon JP, Carpentier A, Donovan D, et al. Integrated obesity care management system implementation and research protocol. BMC Health Serv Res. 2007;7:163. doi: 10.1186/14726963-7-163.

72. Pelletier L, Baillargeon J. Clinically significant and sustained weight loss is achievable in obese women with polycystic ovary syndrome followed in a regular medical practice. Fertil Steril. 2010;94(7):2665-2669. doi: 10.1016/j.fertnstert.2010.02.047. 


\section{Appendices}

Appendix A: IRB Approval and Addendum Approval

Appendix B: Survey Recruitment Distribution Sites

Appendix C: Survey Announcement for List Serves and LinkedIn

Appendix D: Survey Email Request

Appendix E: Survey Email Reminder

Appendix F: Survey

Appendix G: Focus Group Email Invitation

Appendix H: Focus Group Script 


\section{Appendix A: IRB Approval \& Addendum}

WVU Institutional Review Board Approved Protocol H-24546 Title: Multidisciplinary Approach to Treatment of PCOS including Nutrition Therapy on April 4, 2013 and Addendum Approved on January 18, 2014

The 42 page document including consent can be found in the BRAAN 2 system with the tracking number 24546. 


\section{Appendix B: Survey Recruitment Distribution Sites}

\section{ListServs included:}

- Society for Adolescent Health and Medicine (SAHM)

- North American Society for Pediatric and Adolescent Gynecology (NASPAG)

- Society of Assisted Reproductive Technology - American Society of Reproductive Medicine (SART-ASRM)

- EmbryoMail

\section{LinkedIn Groups included:}

- 'Academy of Nutrition and Dietetics'

- 'Endocrinology'

- 'Endocrinology Discussion Network'

- 'Global Physician Assistant Professionals'

- 'Nutrition Entrepreneurs Dietetic Practice Group'

- 'Nutrition Health Providers and Professionals'

- 'Obstetrics \& Gynaecology Networking Group - Women's Health'

- 'Ovarian Club'

- 'Pediatric Endocrinology'

- 'Polycystic Ovary Syndrome Association of Australia'

- 'Registered Dietitian Net'

- 'SCAN: Sports, Cardiovascular, and Wellness Nutrition'

- 'The Endocrine Society'

- 'The Endocrine Society Interest Group'

- 'Weight Management Dietetic Practice Group'

- 'Women Nutritionist' 


\section{Appendix C: Survey Announcement for List Serves and LinkedIn}

\section{DISTRIBUTED VIA LIST SERVES \& LINKED IN}

On behalf of a dietetic intern who compiled a survey to send to individuals/teams for care for patients with PCOS:

Title: Special Request for Individuals Working Closely with PCOS

I am searching for individuals who work with PCOS patients who would be willing to spend about 10 minutes filing out this survey to determine the current trends of PCOS treatment from practitioners. It will also provide insight on future multidisciplinary treatment for patients with PCOS. The West Virginia University Institutional Review Board has approved this research study.

Link to survey: http://wvu.qualtrics.com/SE/?SID=SV_6Vxq8pJ3BZWrRqd

Your participation is greatly appreciated. If you have any questions please contact Wendy Thompson directly at wmthompson@mix.wvu.edu

Wendy Thompson

Graduate Student - Nutrition

West Virginia University

Davis College of Agriculture, Natural Resources, and Design 


\section{Appendix D: Survey Email Request}

\section{SENT TO: "PCOS SURVEY FROM LISTSERV"}

Dear PCOS Practitioner,

Thank you for replying to my request seeking your expertise in PCOS treatment. Below is the link to the survey that you previously expressed interest in taking. This survey should take about 10 minutes to complete. If you are interested in receiving the results please leave your contact information at the end.

Link to survey: http://wvu.qualtrics.com/SE/?SID=SV_6Vxq8pJ3BZWrRqd

The survey will be used to determine the current trends of PCOS treatment across multiple providers around the globe. Your feedback will also provide insight on future multidisciplinary treatment on patients with PCOS. This survey is being conducted by a graduate student in Nutrition at West Virginia University, Wendy Thompson with supervision by Dr. Melissa Olfert. Your participation in this project is greatly appreciated. Should you have any questions, please feel free to contact Wendy Thompson at wmthompson@mix.wvu.edu, or Dr. Melissa Olfert at melissa.olfert@mail.wvu.edu. The West Virginia University Institutional Review Board has approved this research study.

\section{Thank you for your time.}

Sincerely,

Wendy Thompson

WVU Department of Human Nutrition and Foods

Melissa Olfert, DrPH, MS, RD, LD

Assistant Professor - WVU Human Nutrition and Foods 


\section{Appendix E: Survey Email Reminder}

Sent out: $5 / 28 / 13$

\section{SEND TO: "PCOS SURVEY FROM LISTSERV"}

Dear PCOS Practitioner,

Thank you for replying to my request seeking your expertise in PCOS treatment. Below is the link to the survey that you previously expressed interest in taking. If you have not gotten a chance to take it, please take 10 minutes or less to complete the survey before it closes. If you are interested in receiving the results please leave your contact information at the end.

Link to survey: http://wvu.qualtrics.com/SE/?SID=SV_6Vxq8pJ3BZWrRqd

The survey will be used to determine the current trends of PCOS treatment across multiple providers around the globe. Your feedback will also provide insight on future multidisciplinary treatment on patients with PCOS. This survey is being conducted by a graduate student in Nutrition at West Virginia University, Wendy Thompson with supervision by Dr. Melissa Olfert. Your participation in this project is greatly appreciated. Should you have any questions, please feel free to contact Wendy Thompson atwmthompson@mix.wvu.edu, or Dr. Melissa Olfert at melissa.olfert@mail.wvu.edu. The West Virginia University Institutional Review Board has approved this research study.

\section{Thank you for your time.}

Sincerely,

Wendy Thompson

WVU Department of Human Nutrition and Foods

Melissa Olfert, DrPH, MS, RD, LD

$\square$ Assistant Professor - WVU Human Nutrition and Foods 
Appendix F: Survey

\section{W-WestVirginiaUniversity.}

\section{Dear Participant,}

You have been identified as a provider involved with PCOS treatment. Please take about 10 minutes to complete this survey to determine the current trends of PCOS treatment across multiple providers. Your feedback will also provide insight on future multidisciplinary treatment on patients with PCOS. This survey is being conducted by a graduate student in Nutrition at West Virginia University, Wendy Thompson with supervision by Dr. Melissa Olfert. Your participation in this project is greatly appreciated. Should you have any questions, please feel free to contact Wendy Thompson at wmthompson@mix.wvu.edu, or Dr. Melissa Olfert at melissa.olfert@mail.wvu.edu. This research study has been approved by the West Virginia University Institutional Review Board.

Thank you for your time.

Sincerely, Wendy Thompson

WVU Department of Human Nutrition and Foods

Melissa Olfert, DrPH, MS, RD, LD

Assistant Professor - WVU Human Nutrition and Foods 


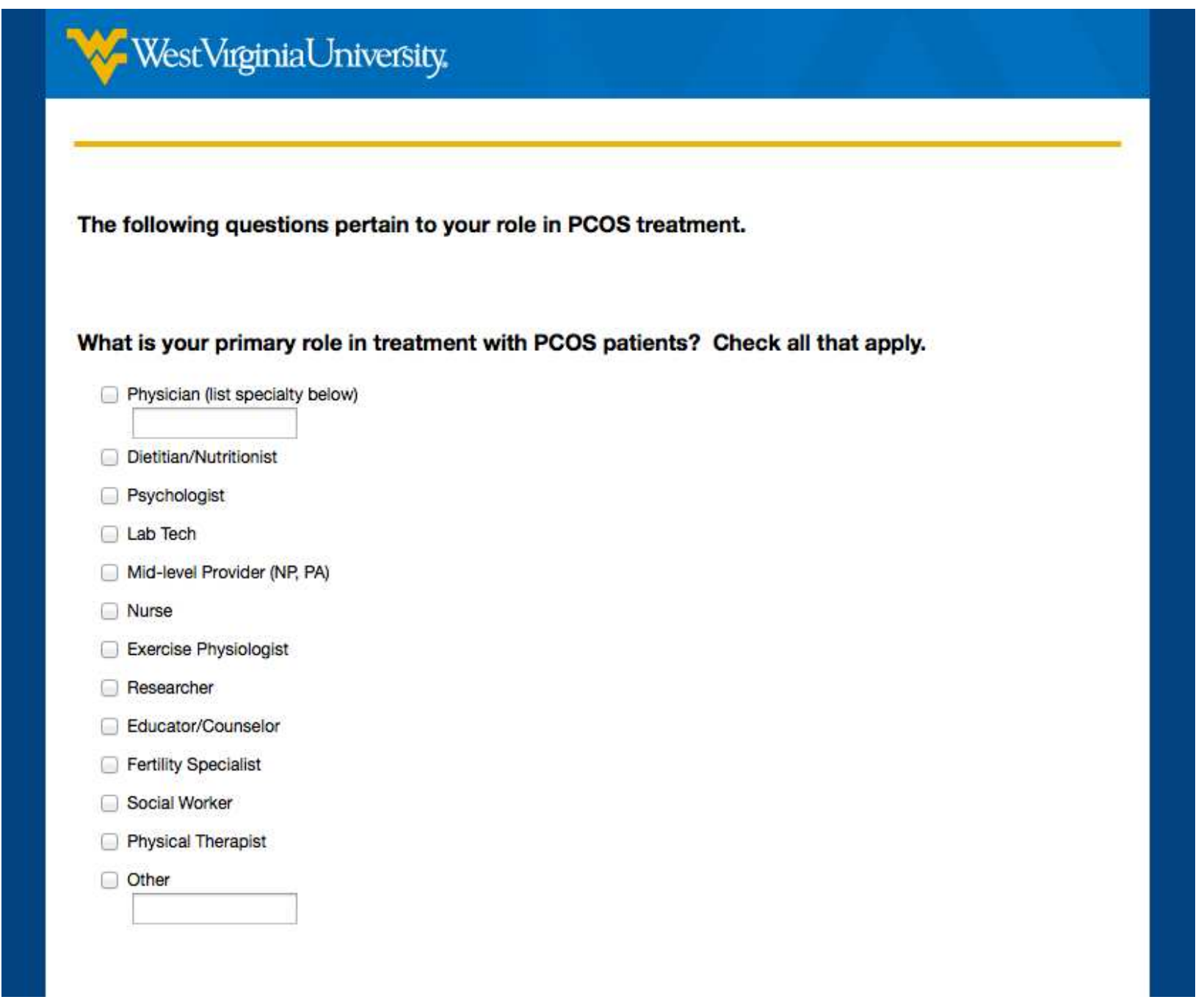


What is your exact title or titles?

Are you male or female?

Male

Female

In what setting do you treat patients with PCOS? Check all that apply.
$\square$ Private Office
$\square$ Hospital/Clinic
Research Facility
$\square$ Other

On average, how many NEW patients do you see for PCOS treatment annually?
$\bigcirc<50$
50-100
100-150
150-200
C $>200$ 
The following questions pertain to the PCOS related facility you are associated with.

What is the name of the facility (i.e. clinic, hospital, university, office) you are affiliated with regarding PCOS treatment?

Is the PCOS related facility named above located in the United States of America?
Yes
No 


\section{W.WestVirginiaUniversity.}

What type of setting is the PCOS related facility located in?
Rural
Urban
Suburban
Other

When did your PCOS related facility first open?
$<2$ years
$2-5$ years ago
5-10 years ago
$>10$ years ago

Does the PCOS related facility you are affiliated with specialize in predominately one type of treatment (i.e. infertility, weight loss, electrophoresis, etc.) or does it treat PCOS comprehensively?

Comprehensive

Do you collect patient satisfaction data?
Yes
No 
WestVirginiaUniversity.

The following questions pertain to the patients you treat in your facility.

What is the average age range of the PCOS patients?

Average Youngest

Average Oldest

Estimate the percentage of your PCOS patients who meet the following criteria for weight status?

Underweight (BMI <18.5)

Normal $(\mathrm{BMI}=18.5-24.9)$

Overweight $(\mathrm{BMI}=25-29.9)$

Obese $(\mathrm{BMI}>30)$

Total 
What is the patient payer provider percentages? If unknown, please leave blank.

\begin{tabular}{|l|l|}
\hline Medicare/Medicaid & 0 \\
\hline Private & 0 \\
\hline Uninsured & 0 \\
\hline Other & 0 \\
\hline Total & 0 \\
\hline
\end{tabular}

What diagnostic criteria do you use to diagnose teenage patients with PCOS?
National Institutes of Health (NIH) Criteria 1990
Rotterdam Criteria 2003
Androgen Excess Society (AES) 2006
Other

What diagnostic criteria do you use to diagnose adult patients with PCOS?

National Institutes of Health (NIH) Criteria 1990

Rotterdam Criteria 2003

Androgen Excess Society (AES) 2006

Other

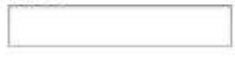

Do you or your referring physician use a screening tool to diagnose patients with PCOS?
Yes
No
Unknown 
WWestVirginiaUniversity.

How are patients typically referred to your PCOS related facility?

Family Physician

Walk-Ins

Other

In an average week, how many TOTAL PCOS patients are seen by any member of the team in your PCOS related facility?
$<<10$
$10-20$
20-30
$30-40$
40-50
$>50$ 


\section{WestVirginiaUniversity.}

The last five questions pertain to future implications for PCOS multidisciplinary clinics.

In your opinion, please rate the following health care providers on how important their role is in a multidisciplinary PCOS clinic.

\begin{tabular}{l|ccccc} 
& Highly Involved & Involved & Neutral & $\begin{array}{c}\text { Occasionally } \\
\text { Involved }\end{array}$ & Never Involved \\
\hline Endocrinologist & 0 & 0 & 0 & 0 & 0 \\
Gynecologist & 0 & 0 & 0 & 0 & 0 \\
Physician (other) & 0 & 0 & 0 & 0 & 0 \\
Dietitian/Nutritionist & 0 & 0 & 0 & 0 & 0 \\
Psychologist & 0 & 0 & 0 & 0 & 0 \\
Mid-level Providers (NP, PA) & 0 & 0 & 0 & 0 \\
Nurse & 0 & 0 & 0 & 0 \\
Exercise Physiologist & 0 & 0 & 0 & 0 \\
Fertility Specialist & 0 & 0 & 0 & 0 \\
Social Worker & 0 & 0 & 0 & 0 \\
Physical Therapist & 0 & 0 & 0 & 0 \\
Other & 0 & 0 & 0 & 0 & 0 \\
Other & 0 & 0 & 0 & 0 & 0 \\
& & & & & 0 \\
\end{tabular}

In your opinion, what are the top one or two items your clinic could improve upon?

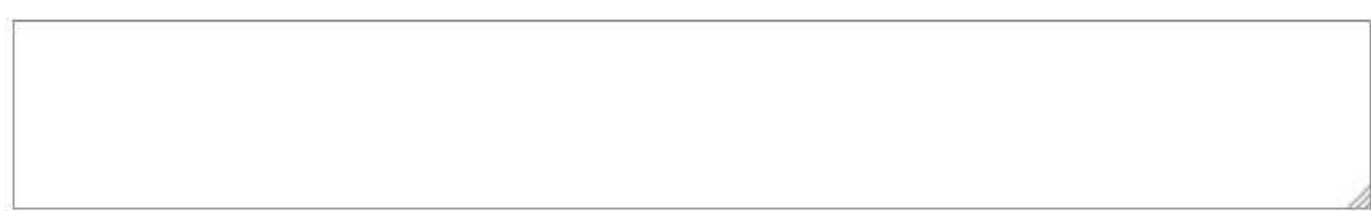

In your opinion, what are the top one or two items that your clinic does well? 
In your opinion, what are the potential barriers that future multidisciplinary PCOS clinics could face?

In your opinion, what are the advantages to patients visiting a multidisciplinary comprehensive PCOS clinic? 
WWestVirginiaUniversity.

Thanks again for your participation. Feel free to contact me at wmthompson@mix.wvu.edu if you have any further questions. If you would like to receive the results of this study please fill out the following information below.

If you know anyone else who would qualify for this survey that might want to take part in this study, please forward on the link.
Name
Address
Address 2
City
State
Postal Code
Country
Email
Phone \# (If in the US)

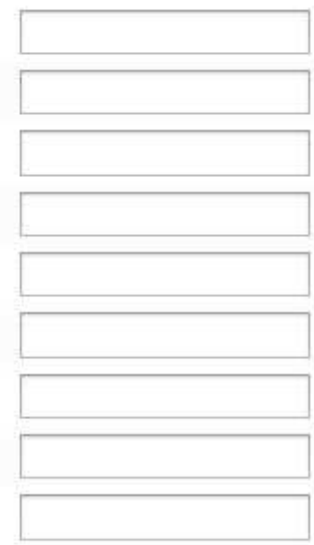




\section{Appendix G: Focus Group Email Invitation}

SENT TO: Survey Respondent's with Contact Information in the US

Dear Participant,

Thank you again for your participation in the 'current trends of PCOS treatment across multiple providers' survey. Your feedback has provided insight on future multidisciplinary treatment on patients with PCOS.

In order to further explore and evaluate the potential future for multidisciplinary PCOS clinics you are being asked to participate in a focus group. The focus group will occur during the first two weeks of February. The duration will be 45 minutes to one hour and will be conducted via telephone. The focus group will be facilitated by a graduate student in Nutrition at West Virginia University, Wendy Thompson with supervision by Dr. Melissa Olfert.

Your participation in this project is greatly appreciated and you will be compensated with a \$25 American Express gift card. If you are interested in participating please respond to this email in the next two weeks and include your profession (i.e. dietitian, physician, etc.).

Should you have any questions, please feel free to contact Wendy Thompson at wmthompson@mix.wvu.edu, or Dr. Melissa Olfert at melissa.olfert@mail.wvu.edu. The West Virginia University Institutional Review Board has approved this research study.

Thanks,

Wendy Thompson

Graduate Dietetic Intern

West Virginia University

Davis College of Agriculture, Natural Resources, and Design

Division of Animal and Nutrition Sciences

Morgantown, WV 26505 


\section{Appendix H: Focus Group Script}

Opening (5 Minutes):

"Hello. My name is Wendy Thompson and I am a current graduate student in Human Nutrition at West Virginia University. I also have two note takers, Jade White and Makenzie Barr and my advisor, Dr. Olfert, here today. The objective of this focus group is to further gather information on the nutrition interventions provided as part of multidisciplinary treatment for patients with PCOS and identify the potential barriers for why dietitians are not more involved. This FG is part of a larger study, which included a survey to better understand multidisciplinary PCOS clinics. Our conversation today will be about 45-60 minutes. Are there any questions?"

Respond to any questions.

"Let's go over a some helpful tips. In order to best keep track of what people are saying let's try to only have one person speaking at a time. Just as a reminder this conversation will be recorded but everything you tell us today will be kept completely confidential. We will summarize the things you tell us and combine it with other focus groups we are administering. Remember there are no right or wrong answers and we want to hear a variety of viewpoints. One of my jobs today, as the moderator, is to make sure we discuss all of the issues we planned to discuss and make sure everyone has a chance to talk."

"Just to get us started, let's have you all tell us your first name and what type of facility you treat patients with PCOS and a simple explanation of your role/title."

"Let's begin."

Questions (40 minutes):

1. Describe any nutritional interventions that you provide to your patients.

a. Prompts:

i. Who provides the nutrition education/counseling?

ii. Do you refer patients to anyone?

iii. What is the extent of the intervention? (i.e. one session only, weekly follow-ups, group settings, doctor recommends weight loss?)

2. How are the dietary intervention decided upon and communicated between providers?

a. Prompts:

i. Is there a protocol or pre-decided approaches?

ii. Does it only focus on weight reduction or does it include other parameters?

iii. For example - macro or micronutrients/special diets?

iv. Is there any "case management" where all team members meet and discuss the patients, their progress, and the care plan, etc. or does everyone do their own thing?

v. Does case management seem to have potentially better outcomes? 
3. When do you think dietary intervention is warranted for a patient with PCOS?

a. Prompts:

i. Age?

ii. Weight category or status? (up to $40 \%$ of patients with PCOS can be of "healthy weight")

iii. What about dietary interventions for individuals of "normal" weight to minimize symptoms and long-term disease risk?

4. In your experience, how accessible are nutritional interventions for the majority of PCOS patients?

a. Prompts:

i. Do you feel that most patients with PCOS are provided with nutritional education or a referral to an $\mathrm{RD}$ ?

ii. Why are some patients not?

1. Does this differ based on weight status?

a. In your opinion, are PCOS patients of normal weight given the same access to nutritional interventions?

iii. After the referral is given, do you think the majority of patients actually see the RD?

5. In your opinion, what are the challenges for getting dietitians more involved with the treatment of PCOS?

a. Prompts:

i. What are some potential reasons why physicians would not provide nutritional education or a referral?

ii. What are potential barriers that would prevent patients from seeing a dietitian - after referral is given?

1. What are potential reasons that they would choose not to?

iii. What are your opinions on the adequacy of evidence-based guidelines for nutrition interventions?

iv. If guidelines were more prevalent - do you feel more dietitians would get involved with PCOS patients?

6. Do you feel like other providers know or understand the value of nutritional inventions for PCOS patients?

a. Prompts:

i. Does it differ by type of provider?

1. Other Physicians?

2. Other Dietitians?

ii. Who do you feel should provide PCOS patients with nutritional counseling?

1. Are they any potential benefits to having an RD provide nutrition counseling over a physician?

a. If so, what are some of those benefits? 
7. In your career with PCOS, do you feel there has been a shift in awareness or interest? a. Prompts:

i. In the medical community?

ii. In the press?

Closure (2 minutes):

"Are there any final questions or comments? (Respond to questions as needed.) Thank you for participating in the focus group today. We really appreciate all of your help and are excited to learn about your thoughts regarding multidisciplinary PCOS treatment and the benefits of nutritional interventions. We will be mailing you the gift card after the call is over so please make sure we have your contact information." 